\title{
FACTORIZATION IN COMMUTATIVE BANACH ALGEBRAS
}

\author{
H. G. DALES, J. F. FEINSTEIN, AND H. L. PHAM
}

\begin{abstract}
Let $A$ be a (non-unital) commutative Banach algebra. We consider when $A$ has a variety of factorization properties: we list the (obvious) implications between these properties, and then consider whether any of these implications can be reversed in various classes of commutative Banach algebras. We summarize the known counter-examples to these possible reverse implications, and add further counter-examples. Some results are used to show the existence of a large family of prime ideals in each non-zero, commutative, radical Banach algebra with a dense set of products.
\end{abstract}

\section{INTRODUCTION}

Let $A$ be a (non-unital) commutative Banach algebra. We wish to examine when $A$ factors in a variety of senses. Our main results are counter-examples to a number of questions that have been raised. Indeed, we shall list seven such factorization properties, called (I)-(VII), and note that each of these immediately implies the next one. We shall also, in $\S 4$, discuss two other 'local' factorization properties, called $(\mathrm{A})$ and $(\mathrm{B})$ (where $(\mathrm{A}) \Rightarrow(\mathrm{B})$ ); these properties are relevant for Esterle's classification of commutative, radical Banach algebras that is given in [14]. We shall then discuss whether or not any of these implications can be reversed when we restrict attention to particular classes of commutative Banach algebras. We shall show that several cannot be reversed, but we leave open other possible reverse implications. A summary in $\S 6$ describes our knowledge at the present time.

We shall concentrate on two particular classes of commutative Banach algebras $A$ : first, on the case where $A$ is semi-simple (so that $A$ is a Banach function algebra), and in particular when $A$ is a maximal ideal in a uniform algebra on a compact space, and, second, on the other extreme case where $A$

2010 Mathematics Subject Classification. Primary 46J10; Secondary 46J15, 46J20, $46 \mathrm{~J} 45$.

Key words and phrases. commutative Banach algebra, Banach function algebra, radical Banach algebra, uniform algebra, approximate identities, peak points, strong boundary points, Choquet boundary, Šilov boundary, factors, factors weakly, factorization of pairs, factors projectively, factors densely, Gleason parts, Volterra algebra, big disc algebra, extensions of uniform algebras, local factorization, Esterle's classification, prime ideals in radical convolution algebras. 
is a radical Banach algebra. We shall also seek results in the special case that our commutative Banach algebra is separable, and when it has the special form of a maximal ideal in a class of uniform algebras that generalize the uniform algebra $R(X)$ for a compact subset $X$ of the complex plane, $\mathbb{C}$; in this latter case most of the stated properties are equivalent to each other.

In the remainder of this introduction, we shall first recall some basic properties of the commutative Banach algebras that we shall consider, and then list our factorization properties (I)-(VII) and note the known implications and counter-examples between them.

In $\S 2$, we shall give some results about factorization for uniform algebras, mainly related to Gleason parts in the character space of the algebra.

In $\S 3$, we shall discuss the theory of extensions of uniform algebras and prove our main theorem that there is a maximal ideal $M$ in a uniform algebra such that null sequences in $M$ factor, but $M$ does not have a bounded approximate identity.

In $\S 4$, we shall introduce the two 'local' factorization properties, called (A) and (B), and discuss their relation with the factorization properties already discussed; in particular, we shall exhibit a separable maximal ideal in a uniform algebra that satisfies condition (B), but not condition (A).

Finally, in $§ 5$, various factorization results will extend Esterle's classification theory of commutative, radical Banach algebras from [14] and will lead to a proof that, for a non-zero, commutative, radical Banach algebra $R$ such that $\{a b: a, b \in R\}$ is dense in $R$, there is a family $\mathcal{F}$ of prime ideals in $R$ with $|\mathcal{F}| \geq \mathfrak{c}$ and such that no member of $\mathcal{F}$ is contained in any other member of $\mathcal{F}$.

For background concerning Banach algebras, see the monograph [6]; however we shall be concerned here only with commutative Banach algebras. (In fact, versions of the properties that we shall discuss do apply to general Banach algebras.) As we said, a particular interest will be the class of uniform algebras; the classic texts on these algebras are those of Browder [3], Gamelin [16], and Stout [33]; more recent texts include [19].

1.1. Notation. We make the following standard definitions:

$\mathbb{I}=[0,1]$ is the closed unit interval in the real line $\mathbb{R}$;

$\mathbb{R}^{+}=\{x \in \mathbb{R}: x \geq 0\}$ and $\mathbb{R}^{+\bullet}=\{x \in \mathbb{R}: x>0\} ;$

$\mathbb{Q}$ is the set of rational numbers; further, $\mathbb{Q}^{+}=\{x \in \mathbb{Q}: x \geq 0\}$ and $\mathbb{Q}^{+\bullet}=\{x \in \mathbb{Q}: x>0\}$; 
$\mathbb{D}=\{z \in \mathbb{C}:|z|<1\}$ is the open unit disc in the complex field, $\mathbb{C}$; and also $\mathbb{T}=\{z \in \mathbb{C}:|z|=1\}$, the unit circle.

For $z \in \mathbb{C}$ and $r>0$, we write $\mathbb{D}(z, r)$ for the open $\operatorname{disc}\{\zeta \in \mathbb{C}:|\zeta-z|<r\}$. For $n \in \mathbb{N}$, we set $\mathbb{N}_{n}=\{1, \ldots, n\}$ and $\mathbb{Z}_{n}^{+}=\{0,1, \ldots, n-1\}$.

The cardinality of a set $S$ is $|S|$; the first infinite ordinal is $\omega$, and the first uncountable ordinal is $\omega_{1}$.

An algebra is always linear and associative and taken over the complex field; the identity of a unital algebra $A$ is $e_{A}$, and the character space of an algebra $A$ is denoted by $\Phi_{A}$. The algebra formed by adjoining an identity, also called $e_{A}$, to a non-unital algebra $A$ is denoted by $A^{\sharp}$ (and $A^{\sharp}=A$ when $A$ is unital). For an algebra $A$, we set $A^{\bullet}=A \backslash\{0\}$.

The (Jacobson) radical of an algebra $A$, defined to be the intersection of the maximal modular left ideals of $A$, is denoted by $\operatorname{rad} A$; it is an ideal in $A$, and the algebra $A$ is semi-simple if $\operatorname{rad} A=\{0\}$ and radical if $A$ has no maximal modular ideals, and so $\operatorname{rad} A=A$. Suppose that $(A,\|\cdot\|)$ is a Banach algebra. Then $\operatorname{rad} A$ is a closed ideal in $A$; when $A$ is commutative,

$$
\operatorname{rad} A=\left\{a \in A: \lim _{n \rightarrow \infty}\left\|a^{n}\right\|^{1 / n}=0\right\} .
$$

Let $A$ be an algebra. An element $a \in A$ is nilpotent if $a^{n}=0$ for some $n \in \mathbb{N}$. For a subset $S$ of $A$ and $n \in \mathbb{N}$, we set

$$
S^{[n]}=\left\{a_{1} \cdots a_{n}: a_{1}, \ldots, a_{n} \in S\right\} \quad \text { and } \quad S^{n}=\operatorname{lin} S^{[n]},
$$

so that $S^{n}$ is the linear span of $S^{[n]}$. The set $S$ is nil if all of its elements are nilpotent, and $S$ is nilpotent if $S^{[n]}=\{0\}$ for some $n \in \mathbb{N}$. (In fact, a Banach algebra that is nil is also nilpotent [6, Theorem 2.6.34].)

The closed unit ball centred at 0 of a normed space $E$ is denoted by $E_{[1]}$ and the dual space of $E$ is $E^{\prime}$, so that $E_{[1]}^{\prime}$ is compact in the weak-* topology, $\sigma\left(E^{\prime}, E\right)$.

Let $E$ and $F$ be Banach spaces. The projective tensor norm $\|\cdot\|_{\pi}$ on $E \otimes F$ is defined by

$$
\|z\|_{\pi}=\inf \left\{\sum_{j=1}^{n}\left\|x_{j}\right\|\left\|y_{j}\right\|: z=\sum_{j=1}^{n} x_{j} \otimes y_{j}, n \in \mathbb{N}\right\} \quad(z \in E \otimes F),
$$

where the infimum is taken over all representations of $z$ as an element of $E \otimes F$. Then $\left(E \otimes F,\|\cdot\|_{\pi}\right)$ is a normed space; the Banach space which is its completion is denoted by $\left(E \widehat{\otimes} F,\|\cdot\|_{\pi}\right)$; it is the projective tensor product of $E$ and $F$.

Let $X$ be a non-empty, locally compact space (always taken to be Hausdorff). The function that is constantly equal to 1 on $X$ is $1_{X}$. We write $C_{0}(X)$ and $C^{b}(X)$ for the spaces of all complex-valued, continuous functions on $X$ 
that vanish at infinity and which are bounded on $X$, respectively, so that $C^{b}(X)$ is a unital algebra with respect to the pointwise algebraic operations and $C_{0}(X)$ is an ideal in $C^{b}(X)$. For $f \in C^{b}(X)$, we write

$$
\mathbf{Z}_{X}(f)=\{x \in X: f(x)=0\},
$$

the zero set of $f$ on $X$. We define

$$
|f|_{X}=\sup \{|f(x)|: x \in X\} \quad\left(f \in C^{b}(X)\right),
$$

so that $|\cdot|_{X}$ is the uniform norm on $X$ and $\left(C^{b}(X),|\cdot|_{X}\right)$ is a commutative, unital Banach algebra. In the case where the space $X$ is compact, we write $C(X)$ for $C_{0}(X)=C^{b}(X)$.

Let $S$ be a non-empty set, and let $E$ be a subset of $\mathbb{C}^{S}$. The weakest topology $\tau$ on $S$ such that each $f \in E$ is continuous with respect to $\tau$ is the E-topology on $S$; it is denoted by $\tau_{E}$.

A function algebra on a non-empty, locally compact space $X$ is a nonzero subalgebra $A$ of $C^{b}(X)$ that separates strongly the points of $X$, in the sense that, for each $x, y \in X$ with $x \neq y$, there exists $f \in A$ with $f(x)=0$ and $f(y)=1$, and is such that the given topology on $X$ is $\tau_{A}$. (In the case where $A$ is a subalgebra of $C_{0}(X)$ that separates strongly the points of $X$, the topology $\tau_{A}$ is necessarily equal to the given topology [6, Proposition 4.1.2].) A Banach function algebra on $X$ is a function algebra $A$ on $X$ with a norm $\|\cdot\|$ such that $(A,\|\cdot\|)$ is a Banach algebra. Suppose that $X$ is compact. Then a uniform algebra on $X$ is a closed subalgebra of $C(X)$ that separates the points of $X$ and contains the constant functions, so that a uniform algebra is a unital Banach function algebra on $X$; such a uniform algebra $A$ is trivial if $A=C(X)$.

For each Banach algebra $A$, the set $\Phi_{A} \cup\{0\}$ is a weak-*-closed subset of $A_{[1]}^{\prime}$, and so $\Phi_{A}$ is locally compact; each Banach function algebra is semisimple and, for each non-zero, commutative, semi-simple Banach algebra $A$, the space $\Phi_{A}$ is non-empty and $A$ is isomorphic (by the Gel'fand transform) to a Banach function algebra on $\Phi_{A}$, and so we can regard $A$ as a Banach function algebra on $\Phi_{A}$ with $A \subset C_{0}\left(\Phi_{A}\right)$.

Let $A$ be a Banach function algebra on a non-empty, locally compact space $X$. The evaluation characters on $A$ are denoted by $\varepsilon_{x}$ for $x \in X$, so that

$$
\varepsilon_{x}(f)=f(x) \quad(f \in A),
$$

and the map $x \mapsto \varepsilon_{x}, X \rightarrow \Phi_{A}$, is a homeomorphic embedding; further, we have $\|f\| \geq|f|_{X} \quad(f \in A)$. The algebra $A$ is natural if each character on $A$ has this form, that is, the above embedding is a surjection, in which case 
$A$ is a subalgebra of $C_{0}(X)$ and each maximal modular ideal of $A$ has the form

$$
M_{x}=\{f \in A: f(x)=0\}=\left\{f \in A: \varepsilon_{x}(f)=0\right\}
$$

for some $x \in X ; M_{x}$ is a Banach function algebra on $X \backslash\{x\}$ whenever $|X| \geq 2$. The Banach function algebras that we shall consider will usually be natural.

For example, let $X$ be a non-empty, compact set in $\mathbb{C}^{n}$, where $n \in \mathbb{N}$. Then $R(X)$ denotes the space of functions in $C(X)$ that are the uniform limits on $X$ of the restrictions to $X$ of functions of the form $p / q$, where $p$ and $q$ are polynomials and $\mathbf{Z}_{X}(q \mid X)=\emptyset$, so that $R(X)$ is a uniform algebra on $X$. The character space of $R(X)$ is identified with the compact subspace of $\mathbb{C}^{n}$ that is the rationally convex hull of $X$. Further, $A(X)$ denotes the uniform algebra of continuous functions on $X$ that are analytic on the interior of $X$, so that $R(X) \subset A(X)$. In the case where $n=1, R(X)$ and $A(X)$ are always natural. See [6, §4.3] and [33, §29], for example.

We shall also consider a class of uniform algebras defined by O'Farrell in [27]. Let $X$ be a non-empty, compact set in $\mathbb{C}$. Then $\mathcal{C}_{X}$ is the class of uniform algebras $A$ on $X$ such that, for each $z \in X$ and each $f \in A$, there is a sequence $\left(g_{n}\right)$ in $C_{0}(\mathbb{C})$ such that, for each $n \in \mathbb{N}, g_{n} \mid X \in A$ and $g_{n}$ is analytic on a neighbourhood of $z$ and such that $\lim _{n \rightarrow \infty} g_{n} \mid X=f$ in $A$. The class $\mathcal{C}_{X}$ clearly contains $R(X)$, and it contains $A(X)$ by a theorem of Arens [6, Lemma 4.3.15]; as in [6, Theorem 4.3.14], each member of $\mathcal{C}_{X}$ is natural on $X$. We shall see in Theorem 2.4 that properties (I)-(VI) that we are considering are mutually equivalent for all maximal ideals in the algebras of this class.

A Banach function algebra $A$ is regular if, for each proper, non-empty, closed subspace $F$ of $\Phi_{A}$ and each $x \in \Phi_{A} \backslash F$, there exists $f \in A$ such that $f(x)=1$ and $f \mid F=0$, and $A$ is normal if, for each non-empty, compact subspace $K$ of $\Phi_{A}$ and each non-empty, closed subspace $F$ of $\Phi_{A}$ with $K \cap F=\emptyset$, there exists $f \in A$ such that $f(x)=1(x \in K)$ and $f(x)=0(x \in F)$. In fact, every regular Banach function algebra is normal (see [6, Proposition 4.1.18(ii)] or [33, Theorem 27.2]).

Let $A$ and $B$ be algebras, and set $\mathfrak{A}=A \otimes B$, the linear space that is the tensor product of $A$ and $B$. Then there is a unique product on $\mathfrak{A}$ with respect to which $\mathfrak{A}$ is an algebra and such that

$$
\left(a_{1} \otimes b_{1}\right)\left(a_{2} \otimes b_{2}\right)=a_{1} a_{2} \otimes b_{1} b_{2} \quad\left(a_{1}, a_{2} \in A, b_{1}, b_{2} \in B\right) .
$$

In the case where $A$ and $B$ are Banach algebras, $\mathfrak{A}$ is a normed algebra with respect to the projective tensor norm $\|\cdot\|_{\pi}$, and its completion 
$\left(A \widehat{\otimes} B,\|\cdot\|_{\pi}\right)$ is a Banach algebra [6, Theorem 2.1.22]. There is a unique bounded linear operator

$$
\pi_{A}: A \widehat{\otimes} A \rightarrow A
$$

such that $\pi_{A}(a \otimes b)=a b(a, b \in A)$, and then $\pi_{A}$ is an algebra homomorphism and $\pi_{A}(A \widehat{\otimes} A)$ is a subalgebra of $A$ and a Banach algebra with respect to the quotient norm from $\left(A \widehat{\otimes} A,\|\cdot\|_{\pi}\right)$.

Let $\left(E_{n}: n \in \mathbb{N}\right)$ be a sequence of non-empty sets such that, for each $n \in \mathbb{N}$, there is a map $\theta_{n}: E_{n+1} \rightarrow E_{n}$. Then $\left(\left(E_{n}, \theta_{n}\right): n \in \mathbb{N}\right)$ is a projective sequence and the projective limit of the sequence is defined to be

$$
\lim \operatorname{proj}\left(E_{n}, \theta_{n}\right)=\left\{\left(x_{n}\right) \in \Pi_{n \in \mathbb{N}} E_{n}: \theta_{n}\left(x_{n+1}\right)=x_{n}(n \in \mathbb{N})\right\} .
$$

The image of the projection from $\lim \operatorname{proj}\left(E_{n}, \theta_{n}\right)$ into $E_{1}$ is denoted by

$$
\lim _{(}\left(E_{n}, \theta_{n}\right) \text {. }
$$

We shall use at some future points the following version of the famous Mittag-Leffler theorem; it is proved by a small variation of the proof given in [6, Corollary A.1.25].

Theorem 1.1. Let $\left(\left(E_{n}, \theta_{n}\right): n \in \mathbb{N}\right)$ be a projective sequence consisting of complete metric spaces $E_{n}$ and continuous maps $\theta_{n}$, and let $X_{n}$ be a subset of $E_{n}$ for each $n \in \mathbb{N}$. Suppose that

$$
X_{n} \subset \overline{\theta_{n}\left(X_{n+1}\right)} \quad(n \in \mathbb{N}) .
$$

Then $X_{1}$ is contained in the closure of $\underset{\longleftarrow}{\lim }\left(E_{n}, \theta_{n}\right)$.

1.2. Factorization properties. In this section we shall delineate the factorization properties that we shall consider. The list of properties has some overlap with a similar list in $[6, \S 2.9$, p. 325].

Our first property related to factorization in commutative Banach algebras involves approximate identities.

Definition 1.2. Let $A$ be a commutative Banach algebra. Then an approximate identity $(\mathrm{AI})$ for $A$ is a net $\left(e_{\nu}\right)$ in $A$ such that $\lim _{\nu} e_{v} a=a$ for each $a \in A$; the $\mathrm{AI}\left(e_{\nu}\right)$ is a bounded approximate identity (BAI) if $\sup _{\nu}\left\|e_{v}\right\|<\infty$, and a contractive approximate identity (CAI) if $\left\|e_{v}\right\| \leq 1$ for each $\nu$.

For example, let $\mathcal{V}$ be the Volterra algebra that is discussed in $[6, \S 4.7]$. Thus $\mathcal{V}$ is the Banach space $\left(L^{1}(\mathbb{I}),\|\cdot\|_{1}\right)$ with the truncated convolution 
product specified by

$$
(f \star g)(t)=\int_{0}^{1} f(s-t) g(t) \mathrm{d} t \quad(t \in \mathbb{I})
$$

for $f, g \in \mathcal{V}$. Then $\mathcal{V}$ is a separable, commutative, radical Banach algebra with a contractive approximate identity.

(I) Let $A$ be a commutative Banach algebra. Then A has property (I) if $A$ has a bounded approximate identity.

Let $A$ be Banach function algebra on a non-empty, locally compact space $X$. Then $x \in X$ is a peak point for $A$ if there exists $f \in A$ such that $f(x)=|f|_{X}=1$ and $|f(y)|<1 \quad(y \in X \backslash\{x\})$, and $x$ is a strong boundary point for $A$ if, for each open neighbourhood $U$ of $x$, there exists $f \in A$ with $f(x)=|f|_{X}=1$ and $|f|_{X \backslash U}<1$. In the case where $X$ is metrizable, every strong boundary point for $A$ is a peak point for $A$.

Let $A$ be a uniform algebra on a non-empty, compact space $X$. Then the set of strong boundary points for $A$ is called the Choquet boundary of $A$, and is denoted by $\Gamma_{0}(A)$. A closed subset $L$ of $X$ is a closed boundary for $A$ if $|f|_{L}=|f|_{X} \quad(f \in A)$; the intersection of all the closed boundaries for $A$ is a closed boundary, called the Šlov boundary, $\Gamma(A)$ [6, Definition 4.3.1(iv)]; by [6, Corollary 4.3.7(i)], $\Gamma(A)=\overline{\Gamma_{0}(A)}$.

The following characterizations of maximal ideals in uniform algebras with property (I) (and several other characterizations) are given in [6, Theorem 4.3.5], save that the implication $(b) \Rightarrow(c)$ is from [9, Theorem 4.1].

Proposition 1.3. Let $A$ be a uniform algebra on a non-empty, compact space $X$, and take $x \in X$ such that $M_{x}$ is non-zero. Then the following conditions on $x$ are equivalent:

(a) $x \in \Gamma_{0}(A)$;

(b) $M_{x}$ has a bounded approximate identity;

(c) $M_{x}$ has a contractive approximate identity.

In particular, we can reformulate property (I) for non-zero maximal ideals in uniform algebras as follows:

(I) Let $M_{x}$ be a non-zero maximal ideal in a uniform algebra $A$. Then $M_{x}$ has property (I) if $x$ is a strong boundary point with respect to $A$.

In [9], a Cole algebra was defined to be a natural uniform algebra $A$ on a non-empty, compact space $X$ such that $\Gamma_{0}(A)=X$. It was a longstanding conjecture, called the 'peak-point conjecture', that $C(X)$ is the only Cole algebra on a compact space $X$. The first counter-example was 
due to Cole [4], and is described in [33, §19]; an example of Basener [1], also described in $[33, \S 19]$, gives a compact space $X$ in $\mathbb{C}^{2}$ such that $R(X)$ is a non-trivial Cole algebra. Further, Feinstein [15] obtained examples of non-trivial, regular Cole algebras on compact, metrizable spaces.

Let $(E,\|\cdot\|)$ be a Banach space. Then a null sequence in $E$ is a sequence $\left(x_{n}\right)$ in $E$ such that $\lim _{n \rightarrow \infty}\left\|x_{n}\right\|=0$; the space of null sequences in $E$ is denoted by $c_{0}(E)$, and $c_{0}(E)$ is itself a Banach space for the norm defined by

$$
\left\|\left(x_{n}\right)\right\|=\sup \left\{\left\|x_{n}\right\|: n \in \mathbb{N}\right\} \quad\left(\left(x_{n}\right) \in c_{0}(E)\right) .
$$

Definition 1.4. Let $A$ be a commutative Banach algebra. Then null sequences in $A$ factor if, for each null sequence $\left(a_{n}\right)$ in $A$, there exist $a \in A$ and a null sequence $\left(b_{n}\right)$ in $c_{0}(A)$ such that $a_{n}=a b_{n}(n \in \mathbb{N})$.

The above condition, from [6, Definition 2.6.11], is important in automatic continuity theory.

(II) Let $A$ be a commutative Banach algebra. Then A has property (II) if null sequences in A factor.

The following result is one form of the famous Cohen's factorization theorem; more general forms of this theorem are given in [6, §2.9].

Theorem 1.5. Let $A$ be a commutative Banach algebra. Suppose that $A$ has a bounded approximate identity. Then null sequences in A factor, and so (I) $\Rightarrow$ (II) for $A$.

Examples of separable Banach function algebras for which null sequences factor, but which do not have a bounded approximate identity, were given by Willis in [36, Examples 3, 5, and 6]; see also [6, Example 2.9.47]. However these examples are not maximal ideals in any uniform algebra. It was shown by Dixon in [12] that a separable Banach algebra for which null sequences factor has an approximate identity. The following theorem will be proved in $\S 3$.

Theorem 1.6. There is a non-zero maximal ideal $M$ in a uniform algebra such that null sequences in $M$ factor, but such that $M$ does not have a bounded approximate identity.

Thus (II) $\nRightarrow$ (I) in the class of maximal ideals of uniform algebras. However, our example $M$ is not separable. Let $M$ be a separable maximal ideal in a uniform algebra such that null sequences in $M$ factor. Then we do not know whether $M$ necessarily has a bounded approximate identity. 
Let $A$ be a commutative Banach algebra, and let $R$ be a non-zero, commutative, radical Banach algebra. Then $A \widehat{\otimes} R$ is also a commutative, radical Banach algebra. Indeed, take elements $a \in A$ and $x \in R$. Then

$$
\left\|(a \otimes x)^{n}\right\|_{\pi}=\left\|a^{n} \otimes x^{n}\right\|_{\pi}=\left\|a^{n}\right\|\left\|x^{n}\right\| \quad(n \in \mathbb{N}),
$$

and hence $a \otimes x \in \operatorname{rad}(A \widehat{\otimes} R)$; the remark then follows because the linear span of the set $\{a \otimes x: a \in A, x \in R\}$ is dense in $A \widehat{\otimes} R$.

Suppose that null sequences in $A$ factor and that $R$ has a bounded approximate identity; for example, we could take $R$ to be the Volterra algebra. Then it is also true that null sequences in the radical Banach algebra $A \widehat{\otimes} R$ factor; this is stated by Willis in [36, p. 619]. We prove this result.

Proposition 1.7. Let $A$ and $B$ be commutative Banach algebras such that null sequences in $A$ factor and such that $B$ has a bounded approximate identity. Then null sequences in $A \widehat{\otimes} B$ factor.

Proof. We may suppose that $A$ and $B$ are non-zero. Take a null sequence $\left(x_{n}: n \in \mathbb{N}\right)$ in $A \widehat{\otimes} B$; we may suppose that $x_{n} \neq 0(n \in \mathbb{N})$.

Take $n \in \mathbb{N}$, and choose $a_{n, i} \in A$ and $b_{n, i} \in B$ for $i \in \mathbb{N}$ such that $x_{n}=\sum_{i=1}^{\infty} a_{n, i} \otimes b_{n, i}$ and

$$
\sum_{i=1}^{\infty}\left\|a_{n, i}\right\|\left\|b_{n, i}\right\|<\left\|x_{n}\right\|_{\pi}+\frac{1}{n}\left\|x_{n}\right\|_{\pi}^{1 / 2} .
$$

We may suppose that $\left\|a_{n, i}\right\|=\left\|x_{n}\right\|_{\pi}^{1 / 2}$ for each $i \in \mathbb{N}$, and then

$$
\sum_{i=1}^{\infty}\left\|b_{n, i}\right\|<\left\|x_{n}\right\|_{\pi}^{1 / 2}+\frac{1}{n}
$$

For each $n \in \mathbb{N}$, choose a sequence $\left(\alpha_{n, i}: i \in \mathbb{N}\right)$ in the interval $[1, \infty)$ such that $\lim _{i \rightarrow \infty} \alpha_{n, i}=\infty$, but such that we still have

$$
\sum_{i=1}^{\infty} \alpha_{n, i}\left\|b_{n, i}\right\|<\left\|x_{n}\right\|_{\pi}^{1 / 2}+\frac{1}{n} .
$$

Next choose a bijection $\sigma: \mathbb{N} \rightarrow \mathbb{N} \times \mathbb{N}$. Then $\left(\alpha_{\sigma(k)}^{-1} a_{\sigma(k)}: k \in \mathbb{N}\right)$ is a null sequence in $A$, and so there exist $a \in A$ and a sequence $\left(u_{n, i}: n, i \in \mathbb{N}\right)$ in $A$ such that $a_{n, i}=a u_{n, i}$ for $n, i \in \mathbb{N}$ and such that $\lim _{k \rightarrow \infty} \alpha_{\sigma(k)}^{-1} u_{\sigma(k)}=0$. On the other hand, $\left(\left(\alpha_{n, i} b_{n, i}: i \in \mathbb{N}\right): n \in \mathbb{N}\right)$ is an element of $S:=c_{0}(E)$, where

$$
E=\left\{\left(y_{i}\right): y_{i} \in B(i \in \mathbb{N}),\left\|\left(y_{i}\right)\right\|_{1}=\sum_{i=1}^{\infty}\left\|y_{i}\right\|<\infty\right\},
$$

so that $E$ is a Banach space and $S$ is a space that is a Banach $B$-module such that $B S$ is dense in $S$. By the version of Cohen's factorization theorem given 
in [6, Theorem 2.9.24], there exist $b \in B$ and $c_{n, i} \in B$ for $n, i \in \mathbb{N}$ such that $\sum_{i=1}^{\infty} \alpha_{n, i}\left\|c_{n, i}\right\|<\infty$ for each $n \in \mathbb{N}$, such that $\lim _{n \rightarrow \infty} \sum_{i=1}^{\infty} \alpha_{n, i}\left\|c_{n, i}\right\|=0$, and also such that $b_{n, i}=b c_{n, i}(n, i \in \mathbb{N})$.

For each $n \in \mathbb{N}$, we see that

$$
\begin{aligned}
\sum_{i=1}^{\infty}\left\|u_{n, i}\right\|\left\|c_{n, i}\right\| & =\sum_{i=1}^{\infty} \alpha_{n, i}^{-1}\left\|u_{n, i}\right\| \alpha_{n, i}\left\|c_{n, i}\right\| \\
& \leq\left(\sup _{k \in \mathbb{N}} \alpha_{\sigma(k)}^{-1}\left\|u_{\sigma(k)}\right\|\right) \sum_{i=1}^{\infty} \alpha_{n, i}\left\|c_{n, i}\right\|<\infty
\end{aligned}
$$

and so we can set

$$
y_{n}=\sum_{i=1}^{\infty} u_{n, i} \otimes c_{n, i} \quad(n \in \mathbb{N})
$$

to obtain a sequence $\left(y_{n}\right)$ in $A \widehat{\otimes} B$. The above calculation also shows that we have $\lim _{n \rightarrow \infty}\left\|y_{n}\right\|_{\pi}=0$, and so $\left(y_{n}\right)$ is a null sequence in $A \widehat{\otimes} B$. Since $x_{n}=(a \otimes b) y_{n}(n \in \mathbb{N})$, it is clear that the null sequence $\left(x_{n}: n \in \mathbb{N}\right)$ factors in $A \widehat{\otimes} B$, as required.

Let $A$ be a non-zero, separable, commutative Banach algebra such that null sequences in $A$ factor, but $A$ does not have a bounded approximate identity, and let $R$ be a non-zero, separable, commutative, radical Banach algebra with a bounded approximate identity (such as the Volterra algebra). Assume that $A \widehat{\otimes} R$ has a bounded approximate identity. Then, by [13, Theorem 8.2], $A$ has a bounded approximate identity, a contradiction. Thus, as in [36], we obtain a separable, commutative, radical Banach algebra in which null sequences factor, but which does not have a bounded approximate identity, and hence (II) $\nRightarrow$ (I) in the class of separable, commutative, radical Banach algebras.

Definition 1.8. Let $A$ be a commutative algebra. Then pairs in $A$ factor if, for each $a_{1}, a_{2} \in A$, there exist $a, b_{1}, b_{2} \in A$ such that $a_{1}=a b_{1}$ and $a_{2}=a b_{2}$.

(III) Let A be a commutative Banach algebra. Then A has property (III) if all pairs in A factor.

Trivially (II) $\Rightarrow$ (III). However, we do not have an example of a commutative Banach algebra that satisfies (III), but not (II).

Definition 1.9. Let $A$ be an algebra. Then $A$ factors if $A=A^{[2]}$ and $A$ factors weakly if $A=A^{2}$.

(IV) Let A be a commutative Banach algebra. Then A has property (IV) if $A$ factors. 
Trivially (III) $\Rightarrow$ (IV).

It was shown by Ouzomgi in [29] that there are maximal ideals $M_{x}$ in the (non-separable) algebra $H^{\infty}(\mathbb{D})$ of all bounded analytic functions on the open unit disc $\mathbb{D}$, regarded as a uniform algebra on its character space $\mathfrak{M}$, such that $M_{x}$ factors, but not all pairs in $M_{x}$ factor, and so (IV) $\nRightarrow$ (III) in the class of maximal ideals of uniform algebras. The point $x$ is such that $\{x\}$ is a one-point Gleason part of $\mathfrak{M}$, as defined below. In fact, for each $f$ in the maximal ideal $M_{x}$, there exist functions $g, h \in M_{x}$ such that $f=g h$ and $|g|_{\mathbb{D}}|h|_{\mathbb{D}}=|f|_{\mathbb{D}}$, and so we have 'controlled factorization' without factorization of pairs. See [22, Chapter 10] and [17] for a study of the character space $\mathfrak{M}$ of $H^{\infty}(\mathbb{D})$.

However, we do not have an example of commutative, radical Banach algebra or of a separable, commutative Banach algebra that satisfies (IV), but not (III). Also, we do not know an example of a maximal ideal in a separable uniform algebra that factors, but does not have a bounded approximate identity.

(V) Let $A$ be a commutative Banach algebra. Then $A$ has property (V) if $A$ factors weakly.

Trivially $(\mathrm{IV}) \Rightarrow(\mathrm{V})$.

Definition 1.10. Let $A$ be a natural Banach function algebra on a locally compact space $X$, and take $x \in X$. A linear functional $d$ on $A$ is a point derivation at $x$ if

$$
d(f g)=f(x) d(g)+g(x) d(f) \quad(f, g \in A) .
$$

Since point derivations at $x$ on $A$ correspond to linear functionals on $M_{x}$ that are zero on $M_{x}^{2}$, it is clear that there are no non-zero point derivations at $x$ if and only if $M_{x}$ factors weakly.

In [36, p. 622], Willis exhibited a separable Banach function algebra $A$ such that every element in $A$ is the sum of two products, and so $A$ factors weakly, but such that $A$ does not factor. Thus $(\mathrm{V}) \nRightarrow(\mathrm{IV})$ in the class of separable Banach function algebras. However, we have no example of a maximal ideal in a uniform algebra or of a commutative, radical Banach algebra with this property.

Example 1.11. We give another interesting example of Willis. (However, the relevant results apply mainly to non-commutative algebras.)

Let $G$ be a locally compact group, and let $L^{1}(G)$ be the corresponding group algebra on $G$. Take a closed ideal $I$ of finite codimension in $L^{1}(G)$. 
In the case where $G$ is amenable, the ideal $I$ has a BAI, and so factors. It is proved in [37] that $I$ always weakly factors, even when it does not have a BAI. For example, let $\mathbb{F}_{2}$ be the free group on two generators. Then the augmentation ideal

$$
\left\{f \in \ell^{1}\left(\mathbb{F}_{2}\right): \sum_{s \in \mathbb{F}_{2}} f(s)=0\right\}
$$

factors weakly, but it seems to be an open question whether it factors.

Definition 1.12. Let $A$ be a Banach algebra. Then $A$ factors projectively if the map $\pi_{A}: A \widehat{\otimes} A \rightarrow A$ is a surjection.

Thus a Banach algebra $A$ factors projectively if and only if there exists $C>0$ such that each $a \in A$ can be written in the form $a=\sum_{n=1}^{\infty} b_{n} c_{n}$, where $b_{n}, c_{n} \in A(n \in \mathbb{N})$ and $\sum_{n=1}^{\infty}\left\|b_{n}\right\|\left\|c_{n}\right\| \leq C\|a\|$. Suppose that $A^{2}$ is dense in $A$. Then $A$ factors projectively if and only if $A$ has the $\pi$-property, as defined in [6, Definition 2.1.26].

(VI) Let $A$ be a commutative Banach algebra. Then A has property (VI) if $A$ factors projectively.

Trivially $(\mathrm{V}) \Rightarrow(\mathrm{VI})$.

We shall now give easy examples of (separable) Banach function algebras and commutative, radical Banach algebras that satisfy (VI), but not (V).

Example 1.13. (i) Take $p$ with $1 \leq p<\infty$, and consider the space $\ell^{p}$ on $\mathbb{N}$, taken with the pointwise product, so that $\ell^{p}$ is a natural Banach function algebra on $\mathbb{N}$ (and the space $\ell^{p}$ is separable). Denote by $\delta_{n}$ the sequence $\left(\delta_{m, n}: m \in \mathbb{N}\right)$. Given $\alpha=\left(\alpha_{n}\right) \in \ell^{1}$, we see that $\alpha=\sum_{n=1}^{\infty}\left(\alpha_{n} \delta_{n}\right) \cdot \delta_{n}$, and so $\ell^{1}$ factors projectively, and hence satisfies (VI).

On the other hand, given $\beta, \gamma \in \ell^{1}$, we have

$$
\sum_{i=1}^{\infty}\left|\beta_{i} \gamma_{i}\right|^{1 / 2} \leq\|\beta\|_{1}^{1 / 2}\|\gamma\|_{1}^{1 / 2}<\infty
$$

by Cauchy-Schwarz, and so $\left(\ell^{1}\right)^{2} \subset \ell^{1 / 2} \subsetneq \ell^{1}$. Thus $\ell^{1}$ does not factor weakly. This shows that (VI) does not imply (V) in the class of separable Banach function algebras.

Similarly, in the case where $1<p<\infty$, the algebra $\ell^{p}$ does not factor projectively, as noted in [6, Example 4.1.42(ii)].

(ii) Now let $V$ be a separable, commutative, radical Banach algebra with a CAI; for example, take $V$ to be the Volterra algebra $\mathcal{V}$. Set $R=\ell^{1}(V)$, 
with coordinatewise product. Then $R$ is also a separable, commutative, radical Banach algebra, and $R$ is a Banach $V$-module with $\overline{V \cdot R}=R$.

We now claim that the Banach algebra $R$ factors projectively. Indeed, take a sequence $u=\left(u_{n}\right) \in R$, where $u_{n} \in V(n \in \mathbb{N})$. By the module form of Cohen's factorization theorem [6, Theorem 2.9.24], there exist $a \in V$ with $\|a\|=1$ and $v=\left(v_{n}\right) \in R$ with $u_{n}=a v_{n}(n \in \mathbb{N})$ and $\|v\| \leq 2\|u\|$. For each $n \in \mathbb{N}$, take $b_{n}$ and $c_{n}$ to be the elements of $R$ that have $a$ and $v_{n}$, respectively, in the $n^{\text {th }}$-coordinate and 0 in all other coordinates. Then $u=\sum_{n=1}^{\infty} b_{n} c_{n}$ in $R$ and

$$
\sum_{n=1}^{\infty}\left\|b_{n}\right\|\left\|c_{n}\right\|=\|a\| \sum_{n=1}^{\infty}\left\|c_{n}\right\|=\|v\| \leq 2\|u\|,
$$

so that $R$ factors projectively.

On the other hand, take elements $\left(u_{n}\right)$ and $\left(v_{n}\right)$ in $R$. Then, as above, we have $\sum_{n=1}^{\infty}\left\|u_{n}\right\|^{1 / 2}\left\|v_{n}\right\|^{1 / 2}<\infty$, and so $R^{2} \subsetneq R$, and hence $R$ does not factor weakly.

This shows that (VI) does not imply (V) in the class of separable, commutative, radical Banach algebras.

However, we do not have an example of a maximal ideal in a uniform algebra that satisfies (VI), but not (V), or even such an ideal that satisfies (VI), but not (IV).

Proposition 1.14. Let $\left(A,\|\cdot\|_{A}\right)$ and $\left(B,\|\cdot\|_{B}\right)$ be Banach algebras such that there is a continuous algebra epimorphism $\theta: A \rightarrow B$. Suppose that $A$ factors projectively. Then $B$ factors projectively.

Proof. There is a constant $C_{1}>0$ such that, for each $b \in B$, there exists $a \in A$ with $\theta(a)=b$ and $\|a\|_{A} \leq C_{1}\|b\|_{B}$. Since $A$ factors projectively, there exists a constant $C_{2}>0$ such that each $a \in A$ is of the form $a=\sum_{n=1}^{\infty} r_{n} s_{n}$, where $r_{n}, s_{n} \in A$ for $n \in \mathbb{N}$ and

$$
\sum_{n=1}^{\infty}\left\|r_{n}\right\|_{A}\left\|s_{n}\right\|_{A} \leq C_{2}\|a\|_{A} .
$$

Take $b \in B$, choose $a \in A$ as above, and set $x_{n}=\theta\left(r_{n}\right)$ and $y_{n}=\theta\left(s_{n}\right)$ in $B$ for each $i \in \mathbb{N}$. Then $b=\sum_{n=1}^{\infty} x_{n} y_{n}$, and

$$
\sum_{n=1}^{\infty}\left\|x_{n}\right\|_{B}\left\|y_{n}\right\|_{B} \leq C_{2}\|\theta\|^{2}\|a\|_{A} \leq C_{1} C_{2}\|\theta\|^{2}\|b\|_{B},
$$

and so $B$ factors projectively. 
Proposition 1.15. Let $A$ be a Banach algebra. Suppose that there are a dense subset $S$ of $A$ and $C>0$ such that, for each $a \in S$, there exist $b, c \in A$ with $a=b c$ and $\|b\|\|c\| \leq C\|a\|$. Then $A$ factors projectively.

Proof. Take $a_{0} \in A^{\bullet}$, say $\left\|a_{0}\right\|<1$. First, choose $b_{1}, c_{1} \in A$ such that $\left\|a_{0}-b_{1} c_{1}\right\|<1 / 2$ and $\left\|b_{1}\right\|\left\|c_{1}\right\| \leq C$, and set $a_{1}=a_{0}-b_{1} c_{1}$, so that $\left\|a_{1}\right\|<1 / 2$. Now take $n \in \mathbb{N}$, and assume inductively that we have chosen $b_{1}, \ldots, b_{n}, c_{1}, \ldots, c_{n} \in A$ such that

$$
\left\|a_{0}-\sum_{i=1}^{n} b_{i} c_{i}\right\|<\frac{1}{2^{n}} \quad \text { and } \quad\left\|b_{i}\right\|\left\|c_{i}\right\| \leq \frac{C}{2^{i-1}} \quad\left(i \in \mathbb{N}_{n}\right) .
$$

Set $a_{n}=a_{0}-\sum_{i=1}^{n} b_{i} c_{i}$, so that $\left\|a_{n}\right\|<1 / 2^{n}$, and then choose $b_{n+1}, c_{n+1} \in A$ such that

$$
\left\|a_{n}-b_{n+1} c_{n+1}\right\|<\frac{1}{2^{n+1}} \quad \text { and } \quad\left\|b_{n+1}\right\|\left\|c_{n+1}\right\| \leq \frac{C}{2^{n}} .
$$

The inductive construction continues.

Now $a_{0}=\sum_{i=1}^{\infty} b_{i} c_{i}$ and $\sum_{i=1}^{\infty}\left\|b_{i}\right\|\left\|c_{i}\right\| \leq 2 C$, and so $a_{0} \in \pi_{A}(A \widehat{\otimes} A)$. Thus $A$ factors projectively.

Let $T$ be a sub-semigroup of $\left(\mathbb{R}^{+},+\right)$, and take $\omega$ to be a weight on $T$, so that $\omega(t)>0(t \in T)$ and

$$
\omega(s+t) \leq \omega(s) \omega(t) \quad(s, t \in T) .
$$

The point mass at $t \in T$ is denoted by $\delta_{t}$. Then $\ell^{1}(T, \omega)$ consists of the elements

$$
f=\sum_{t \in T} \alpha_{t} \delta_{t} \quad \text { such that } \quad\|f\|_{\omega}=\sum_{t \in T}\left|\alpha_{t}\right| \omega(t)<\infty ;
$$

this space is a commutative Banach algebra with respect to convolution multiplication $\star$. The weight $\omega$ is radical if $\lim _{n \rightarrow \infty}\left\|\delta_{n t}\right\|_{\omega}^{1 / n}=0(t \in T)$, in which case $\left(\ell^{1}(T, \omega), \star\right)$ is a commutative, radical Banach algebra; it is separable whenever $T$ is countable.

A sub-semigroup $T$ of $\mathbb{R}^{+\bullet}$ is a difference sub-semigroup if $t-s \in T$ whenever $s, t \in T$ and $t>s$.

Corollary 1.16. Let $T$ be a difference sub-semigroup of $\mathbb{R}^{+\bullet}$ such that $\inf T=0$, and let $\omega$ be a continuous weight on $\mathbb{R}^{+}$. Then the semigroup algebra $\left(\ell^{1}(T, \omega), \star\right)$ factors projectively.

Proof. Set $A=\left(\ell^{1}(T, \omega), \star\right)$, and take $S$ be the subalgebra of $A$ consisting of finite linear combinations of point masses, so that $S$ is dense in $A$. For each $f=\sum_{i=1}^{n} \alpha_{i} \delta_{t_{i}} \in S$, choose $t \in(0,1) \cap T$ such that $t<t_{i}$, so that $t_{i}-t \in T$, and such that $\omega\left(t_{i}-t\right) \leq 2 \omega\left(t_{i}\right)$ for each $i \in \mathbb{N}_{n}$, and then 
set $g=\delta_{t}$ and $h=\sum_{i=1}^{n} \alpha_{i} \delta_{t_{i}-t}$. Then $g, h \in A$ with $f=g \star h$, and $\|g\|\|h\| \leq C\|f\|$, where $C=2 \sup \{\omega(t): 0 \leq t \leq 1\}$. By Proposition 1.15, $A$ factors projectively.

Corollary 1.17. Let $M$ be a maximal ideal in a uniform algebra, and suppose that $\left\{f^{2}: f \in M\right\}$ is dense in $M$. Then $M$ factors projectively.

Proof. Set $S=\left\{f^{2}: f \in M\right\}$. Then $S$ satisfies the conditions specified in Proposition 1.15, and so $M$ factors projectively.

Example 1.18. We claim that there is a separable uniform algebra with a maximal ideal $M$ such that $M$ factors projectively, but such that there are pairs in $M$ that do not factor, so that (VI) $\nRightarrow$ (III) in the class of maximal ideals in separable uniform algebras.

Indeed, take $N$ to be a maximal ideal in a uniform algebra on a compact space $X$ such that $N$ has controlled factorization, but does not have factorization of (some) pairs. Such an example (in $\left.H^{\infty}(\mathbb{D})\right)$ has already been mentioned on page 11 .

We shall give an inductive construction.

First, take $f_{1}$ and $f_{2}$ in $N$ such that the pair $\left\{f_{1}, f_{2}\right\}$ does not have a common factor in $N$, and set $S_{0}=\left\{f_{1}, f_{2}\right\}$, and then take $T_{0}$ to be the algebra over the field $\mathbb{Q}+\mathrm{i} \mathbb{Q}$ generated by $f_{1}$ and $f_{2}$, so that $T_{0}$ is a countable set.

Next take $n \in \mathbb{Z}^{+}$, and assume that we have constructed a countable set $S_{n}$ in $N$ and also the space $T_{n}$ which is the algebra over $\mathbb{Q}+\mathrm{i} \mathbb{Q}$ generated by $S_{n}$, so that $T_{n}$ is countable and $T_{n} \subset N$. For each $f \in T_{n}$, take $g, h \in N$ with $f=g h$ and $|g|_{X}|h|_{X}=|f|_{X}$, and let $S_{n+1}$ be the union of $T_{n}$ and all these new functions $g$ and $h$, so that $S_{n+1}$ is also countable. Again take $T_{n+1}$ to be the algebra over $\mathbb{Q}+\mathrm{i} \mathbb{Q}$ generated by the set $S_{n+1}$. This continues the inductive construction.

Define $S=\bigcup\left\{S_{n}: n \in \mathbb{N}\right\}=\bigcup\left\{T_{n}: n \in \mathbb{N}\right\}$, a countable subset of $N$ and an algebra over $\mathbb{Q}+\mathrm{i} \mathbb{Q}$, and define $M$ to be the closure of $S$ in $N$, so that $M$ is a closed subalgebra of $N$ and $M$ is separable. We may regard $A=M^{\sharp}$ as a uniform algebra on a compact space, say $Y$, which is a quotient space of $X$, and $M$ is a maximal ideal in $A$. Further, for each $f \in S$, there exist $g, h \in S$ with $f=g h$ and $|g|_{Y}|h|_{Y}=|f|_{Y}$. Thus $S$ satisfies the conditions in Proposition 1.15 (with respect to $M$ ), and so, by that proposition, $M$ factors projectively. 
On the other hand, it cannot be that the pair $\left\{f_{1}, f_{2}\right\}$ has a common factor in $M$ because it does not have a common factor in $N$.

There is a class of separable, commutative Banach algebras that, at first sight, might give examples that separate the classes satisfying the properties (I) - (VI), and we describe the algebras in this class.

Indeed, a function $\omega: \mathbb{R}^{+\bullet} \rightarrow \mathbb{R}^{+\bullet}$ is a weight function if it is Lebesgue measurable and if

$$
\omega(s+t) \leq \omega(s) \omega(t)\left(s, t \in \mathbb{R}^{+\bullet}\right) .
$$

We denote by $L^{1}(\omega)$ the set of complex-valued, measurable functions on $\mathbb{R}^{+\bullet}$ such that

$$
\|f\|_{\omega}=\int_{0}^{\infty}|f(t)| \omega(t) \mathrm{d} t<\infty
$$

so that $\left(L^{1}(\omega),\|\cdot\|_{\omega}\right)$ is a commutative Banach algebra with respect to the convolution product $\star$.

However the hope of finding counter-examples in this class is dashed by the following result of Ouzomgi [28]. In fact, in [28], only the equivalence of clauses (a) - (d) in the following proposition is stated, but the arguments given also show that $(\mathrm{e}) \Rightarrow(\mathrm{a})$. Here $m$ denotes Lebesgue measure on $\mathbb{R}^{+} \bullet$.

Proposition 1.19. Let $\omega$ be a weight function on $\mathbb{R}^{+\bullet}$. Then the following conditions on $\omega$ are equivalent:

(a) there exists $M>0$ such that $m(\{t \in[0, \delta]: \omega(t)<M\})>0$ for each $\delta>0$

(b) $L^{1}(\omega)$ has a bounded approximate identity;

(c) $L^{1}(\omega)$ factors;

(d) $L^{1}(\omega)$ factors weakly;

(e) $L^{1}(\omega)$ factors projectively.

Thus properties (I) - (VI) are equivalent for the convolution algebras $L^{1}(\omega)$.

Example 1.20. Take $A=\ell^{1}\left(\mathbb{Q}^{+\bullet}, \omega\right)$ for a continuous weight $\omega$ on $\mathbb{R}^{+}$. Then $A$ satisfies (VI) by Corollary 1.16 . However $A$ does not satisfy (III). To see this, first note that it is a consequence of Titchmarsh's convolution theorem [6, Theorem 4.7.22] that any factorization of $\delta_{1}$ in $A$ has the form

$$
\delta_{1}=\left(\alpha \delta_{s}+f\right) *\left(\beta \delta_{t}+g\right),
$$

where $s, t>0$ and $s+t=1$, where $\alpha, \beta \in \mathbb{C}$ with $\alpha \beta=1$, and where $f$ and $g$ have supports contained in $(s, \infty)$ and $(t, \infty)$, respectively. On the other 
hand, each element $h:=\sum_{n=1}^{\infty} \alpha_{n} \delta_{1 / n}$ in $A$, where $\alpha_{n} \neq 0(n \in \mathbb{N})$, cannot have as a factor any element of the form $\alpha \delta_{s}+f$, as described, and so the pair $\left\{\delta_{1}, h\right\}$ has no common factor in $A$.

By taking $\omega$ to be a radical weight, we obtain a separable, commutative, radical Banach algebra $R=\ell^{1}\left(\mathbb{Q}^{+\bullet}, \omega\right)$ that satisfies (VI), but not (III).

It seems unlikely that such a commutative, radical Banach algebra $R$ factors or factors weakly, but we do not know this.

A further class of Banach function algebras that we consider is the following. Let $\Gamma$ be a locally compact group, and take $p$ with $1<p<\infty$. Then the Figà-Talamanca-Herz algebra on $\Gamma$ is $A_{p}(\Gamma)$ : this algebra is described in [6, pp. 493-494] and in the book of Derighetti [11]. The Fourier algebra $A(\Gamma)$ is the algebra $A_{2}(\Gamma)$; see the recent book of Kaniuth and Lau [24]. The algebra $A_{p}(\Gamma)$ is a natural, self-adjoint, translation-invariant, regular Banach function algebra on $\Gamma$. We enquire when $A_{p}(\Gamma)$ has the properties that we are considering.

The following is taken from [25, Proposition 2]; the equivalence of (a) and (b) is also given in [32, Theorem 10.4]

Theorem 1.21. Let $\Gamma$ be a locally compact group, and take $p$ such that $1<p<\infty$. Then the following are equivalent:

(a) the group $\Gamma$ is amenable;

(b) $A_{p}(\Gamma)$ has a bounded approximate identity;

(c) $A_{p}(\Gamma)$ factors weakly.

Thus properties $(\mathrm{I})-(\mathrm{V})$ are equivalent for the Figà-Talamanca-Herz algebras, $A_{p}(\Gamma)$. It seems likely that property (VI) is also equivalent to these properties; towards this, we note the following theorem. Here $\mathbb{F}_{2}$ is the free group on two generators; of course, $\mathbb{F}_{2}$ is not amenable.

Theorem 1.22. Take $p$ such that $1<p<\infty$. Then $A_{p}\left(\mathbb{F}_{2}\right)$ does not factor projectively.

Before giving the proof of this theorem, we recall some standard background that applies to any discrete group $\Gamma$, with identity $e$.

By [11, Definition 2, §4.1], $P M_{p}(\Gamma)$, the space of $p$-pseudo-measures on $\Gamma$, is the closure of $\ell^{1}(\Gamma)$ in $\mathcal{B}\left(\ell^{p}(\Gamma)\right)$ in the ultraweak topology. In fact, $P M_{p}(\Gamma)=A_{p}(\Gamma)^{\prime}$, and the duality is given by

$$
\left\langle\sum_{i=1}^{\infty} g_{i} \star h_{i}, T\right\rangle=\sum_{i=1}^{\infty}\left\langle T \check{g}_{i}, h_{i}\right\rangle,
$$


where $\check{g}(y)=g\left(y^{-1}\right) \quad(y \in \Gamma)$. By [11, Theorem 6, §4.1], this ultraweak topology is the same as the weak-* topology, $\sigma\left(A_{p}(\Gamma)^{\prime}, A_{p}(\Gamma)\right)$. Thus, for $f \in A_{p}(\Gamma)$, we have

$$
\|f\|_{A_{p}(\Gamma)}=\sup \left\{\left|\left\langle f, T_{g}\right\rangle\right|: g \in \ell^{1}(\Gamma),\left\|T_{g}\right\|_{P M_{p}(\Gamma)} \leq 1\right\} .
$$

In the above supremum, we can suppose that $g$ has finite support.

Let $E$ be a Leinert set in $\Gamma$. This means that the restriction algebra

$$
A(E)=\{f \mid E: f \in A(\Gamma)\}
$$

(with the quotient norm) is isomorphic to $\ell^{2}(\mathbb{N})$ as a Banach algebra. Then it is shown in clause (b) of the proof of [2, Proposition 1] that, for such a set $E$ and for each $p$ with $1<p \leq 2$, there is a constant $C_{p}$ such that

$$
\|f \star g\|_{p} \leq C_{p}\|f\|_{p}\|g\|_{p} \quad\left(f \in \ell^{p}(E), g \in \ell^{p}(\Gamma)\right) .
$$

Thus, for each $f \in \ell^{p}(E)$, we have $\left\|T_{f}\right\|_{P M_{p}(\Gamma)} \leq C_{p}\|f\|_{p}$. On the other hand, we have $\left\|T_{f}\right\|_{P M_{p}(\Gamma)} \geq\left\|f \star \delta_{e}\right\|_{p}=\|f\|_{p}$.

We now claim that, for a Leinert set $E$ in $\Gamma$ and $1<p \leq 2$, the restriction map

$$
R: A_{p}(\Gamma) \rightarrow \ell^{\infty}(E)
$$

is such that $\|R(f)\|_{q} \leq C_{p}\|f\|_{A_{p}(\Gamma)}\left(f \in A_{p}(\Gamma)\right)$, where $q=p^{\prime}$, the conjugate index to $p$. This claim says that the map $R: A_{p}(\Gamma) \rightarrow \ell^{q}(E)$ is a bounded linear operator. For this, take $f \in A_{p}(\Gamma)$. It follows from equations (1.2) and (1.3) that

$$
\|f\|_{A_{p}(\Gamma)} \geq \frac{1}{C_{p}} \sup \left\{\left|\sum_{x \in E} f(x) g(x)\right|\right\},
$$

where now the supremum is taken over all functions $g$ with finite support in $E$ such that $\|g\|_{p} \leq 1$. This implies that $\|R(f)\|_{q} \leq C_{p}\|f\|_{A_{p}(\Gamma)}$, giving the claim.

Since $\ell^{q}(\Gamma)$ is a subset of $A_{p}(\Gamma)$, the map $R: A_{p}(\Gamma) \rightarrow \ell^{q}(E)$ is a surjection. It now follows that $\ell^{q}(E)$ is a quotient of $A_{p}(\Gamma)$.

A subset $E$ of $\Gamma$ satisfies Leinert's condition if, for each $n \in \mathbb{N}$ and each $x_{1}, \ldots, x_{2 n} \in \Gamma$ with $x_{i} \neq x_{i+1}(i=1, \ldots, 2 n-1)$, necessarily $x_{1} x_{2}^{-1} \cdots x_{2 n-1} x_{2 n}^{-1} \neq e$. It was shown by Leinert in [23] that every subset $E$ of $\Gamma$ that satisfies this condition is a Leinert set. It follows immediately that $\mathbb{F}_{2}$ contains a Leinert set.

Proof of Theorem 1.22 First suppose that $1<p \leq 2$. Let $E$ be a Leinert set in $\mathbb{F}_{2}$. Then we have shown above that there is a quotient map from $A_{p}\left(\mathbb{F}_{2}\right)$ onto $\ell^{q}(E)$, where $q=p^{\prime}$. The latter Banach algebra is isomorphic 
to $\ell^{q}(\mathbb{N})$, and we have noted in Example 1.13 that $\ell^{q}(\mathbb{N})$ does not factor projectively. Thus, by Proposition $1.14, A_{p}\left(\mathbb{F}_{2}\right)$ does not factor projectively.

Now suppose that $2 \leq p<\infty$. Since the map $f \mapsto \check{f}, A^{p}(\Gamma) \rightarrow A^{q}(\Gamma)$, is an isometric algebra isomorphism, the result again follows.

The following theorem is a slight extension of Theorem 1.22.

Theorem 1.23. Let $\Gamma$ be a locally compact group that contains $\mathbb{F}_{2}$ as a closed subgroup, and take $p$ such that $1<p<\infty$. Then $A_{p}(\Gamma)$ does not factor projectively.

Proof. By a theorem of Herz ([21, Theorem 1]; see also [11, Theorem 5 of $\S 7.9])$, the restriction map $F \mapsto F \mid \mathbb{F}_{2}$ is a continuous algebra epimorphism from $A_{p}(\Gamma)$ onto $A_{p}\left(\mathbb{F}_{2}\right)$. It follows from Proposition 1.14 and Theorem 1.22 that $A_{p}(\Gamma)$ does not factor projectively.

The final statement about factorization in commutative Banach algebras that we shall consider is the following.

Definition 1.24. Let $A$ be a Banach algebra. Then $A$ factors densely if $A^{2}$ is dense in $A$.

(VII) Let A be a commutative Banach algebra. Then A has property (VII) if $A$ factors densely.

Trivially (VI) $\Rightarrow$ (VII).

Let $A$ be a natural Banach function algebra on a non-empty, locally compact space $X$, and take $x \in X$. It is clear that there are no non-zero, continuous point derivations at $x$ if and only if $M_{x}$ factors densely.

There are many non-zero, commutative, radical Banach algebras that do not factor densely: the extreme case is a non-zero Banach space with the zero product.

In Example 2.7 below, we shall show that $(\mathrm{VII}) \not(\mathrm{VI})$ in the class of maximal ideals of separable uniform algebras; we now show that (VII) $\nRightarrow$ (VI) in the class of separable, commutative, radical Banach algebra.

Example 1.25. Consider $R=C_{*, 0}(\mathbb{I})$, the algebra of all continuous functions on $\mathbb{I}$ that vanish at 0 , taken with the truncated convolution product given in equation (1.1) (see [6, Definition 4.7.39]), so that $R$ is a radical Banach algebra. Then $R$ has an approximate identity, and so $\overline{R^{[2]}}=R$, and hence $R$ satisfies (VII). But $R$ fails to satisfy (VI). For suppose that $f, g \in R$. Then $|(f * g)(t)| \leq|f|_{\mathbb{I}}|g|_{\mathbb{I}} t(t \in \mathbb{I})$, and so each element $f \in \pi_{R}(R \widehat{\otimes} R)$ is such that $f(t)=O(t)$ as $t \rightarrow 0+$, and this is not true for every $f \in R$. 


\section{Gleason Parts}

Here we shall recall the standard theory of Gleason parts for a uniform algebra, and give some examples.

Let $A$ be a natural uniform algebra on a non-empty, compact space $X$, and take $x, y \in X$. Then the following are equivalent:

(a) $\left\|\varepsilon_{x}-\varepsilon_{y}\right\|<2$;

(b) there exists $c \in(0,1)$ such that $|f(x)| \leq c|f|_{X} \quad\left(f \in M_{y}\right)$.

See [33, §16], where several other equivalent conditions are given. In the case where the conditions hold, we define $x \sim y$. It is standard that $\sim$ is an equivalence relation on $X$; the equivalence classes are the Gleason parts of $X$ (with respect to $A$ ). These parts form a partition of $X$, and each part is a completely regular and $\sigma$-compact topological space with respect to the Gel'fand topology; by a theorem of Garnett, these are the only topological restrictions on Gleason parts.

For a discussion of Gleason parts, including Garnett's theorem, see [16, Chapter VI], [17, Chapter X], and [33, §18].

A contractive pointwise approximate identity (CPAI) in a natural Banach function algebra $A$ on a non-empty, locally compact space $X$ is a net $\left(f_{\nu}\right)$ in $A_{[1]}$ such that

$$
\lim _{\nu} f_{\nu}(x)=1 \quad(x \in X)
$$

the Banach function algebra $A$ is pointwise contractive if each non-zero, maximal modular ideal in $A$ has a contractive pointwise approximate identity. For a classification of pointwise contractive Banach function algebras that have a 'BSE norm', see [9]; further theory of contractive pointwise approximate identities is given in [10]. The relevance of this for us is the following result from [9, Theorem 4.6].

Proposition 2.1. Let $A$ be a natural uniform algebra on a compact space $X$, and take $x \in X$ such that $M_{x}$ is non-zero. Then $\{x\}$ is a one-point Gleason part if and only if $M_{x}$ has a contractive pointwise approximate identity.

Let $A$ be a natural uniform algebra on a compact space $X$. Certainly $\{x\}$ is a one-point part whenever $x \in X$ is a strong boundary point for $A$. However, there are several examples where $\{x\}$ is a one-point Gleason part with respect to $A$, but $x$ is not a strong boundary point for $A$; see $[33, \S 19]$. In particular, this is the case for the big disc algebra, described below. As noted above, there are maximal ideals $M_{x}$ in the (non-separable) uniform algebra $H^{\infty}(\mathbb{D})$ that factor when $\{x\}$ is a one-point part that is 
not a strong boundary point; it is shown in [18] that there can be points $x$ in the character space $X$ of a (non-separable) uniform algebra $A$ such that $M_{x}$ factors, but $\{x\}$ is not a one-point Gleason part. On the other hand, it is not true that $M_{x}$ factors whenever $\{x\}$ is a one-point part; stronger versions of this fact will be noted at the end of this section.

The following proof extends ones in [4, Lemma 1.1(i)] and in [33, p. 201].

Proposition 2.2. Let $A$ be a natural uniform algebra on a compact space $X$, and take $x \in X$. Suppose that $\left\{f^{2}: f \in M_{x}\right\}$ is dense in $M_{x}$. Then $\{y\}$ is a one-point Gleason part for each $y \in X$.

Proof. We may suppose that $|X| \geq 2$.

Let $S_{n}=M_{x}(n \in \mathbb{N})$, and define $\theta_{n}: f \mapsto f^{2}, S_{n+1} \rightarrow S_{n}$, for $n \in \mathbb{N}$ to obtain a projective sequence. The maps $\theta_{n}$ are continuous and have dense range, and so, by Theorem 1.1, $S$ is dense in $M_{x}$, where $S=\lim \left(S_{n}, \theta_{n}\right)$. Clearly, for each $f \in S$ and $k \in \mathbb{N}$, there exists $g \in S$ with $g^{2^{k}}=f$.

Take $y \in X \backslash\{x\}$ and $\varepsilon>0$. Then there exists $f \in S_{[1]}$ with $|f(y)| \neq 0$. Take $k \in \mathbb{N}$ with $|f(y)|>(1-\varepsilon)^{2^{k}}$, and then take $g \in S_{[1]}$ with $g^{2^{k}}=f$. Thus $|g(y)|>1-\varepsilon$, and so $y \not x$.

Now take $y, z \in X \backslash\{x\}$, choose $f \in M_{x}$ with $f(y)=0$ and $f(z)=1$, and fix $\varepsilon \in(0,1)$. Choose $n \in \mathbb{N}$ with $1+|f|_{X}<(1+\varepsilon)^{2^{n}}$ and $(1-\varepsilon)^{2^{n}}<\varepsilon$, and then choose $\eta>0$ with $\eta<\varepsilon^{2^{n}}$ and $\varepsilon+\eta<1$. There exists $g \in S$ with $\left|f-g^{2^{n}}\right|_{X}<\eta$. Then $|g|_{X}^{2^{n}}<(1+\varepsilon)^{2^{n}}$, and so $|g|_{X}<1+\varepsilon$. Also $|g(y)|<\varepsilon$.

Assume that $|g(z)|<1-\varepsilon$. Then $|g(z)|^{2^{n}}<\varepsilon$, and so $|f(z)|<\varepsilon+\eta<1$, a contradiction. Thus $|g(z)| \geq 1-\varepsilon$.

Finally, set $h=\left(g-g(y) 1_{X}\right) /(1+\varepsilon)$. Then $h \in M_{y}$ and $|h|_{X} \leq 1$, and we also have $|h(z)| \geq(1-2 \varepsilon) /(1+\varepsilon)$, and so $y \not z$

It follows that $\{y\}$ is a one-point part for each $y \in X$.

The following result is essentially [3, Theorem 1.6.2].

Proposition 2.3. Let $A$ be a natural uniform algebra on a non-empty, compact space $X$, and take $x \in X$. Suppose that $M_{x}$ factors projectively. Then $x$ is an isolated point with respect to the Gleason metric on $X$.

Proof. We may suppose that $|X| \geq 2$.

Set $M=M_{x}$. The map $\pi_{M}: M \widehat{\otimes} M \rightarrow M$ is a surjection, and so, by the open mapping theorem, there exists $m>0$ such that, for each $f \in M_{[1]}$, there exist $f_{j}, g_{j} \in M_{[1]}$ and $\alpha_{j}>0$ for $j \in \mathbb{N}$ such that

$$
f-f(x)=\sum_{j=1}^{\infty} \alpha_{j} f_{j} g_{j}=\sum_{j=1}^{\infty} \alpha_{j}\left(f_{j}-f_{j}(x)\right)\left(g_{j}-g_{j}(x)\right)
$$


and $\sum_{j=1}^{\infty} \alpha_{j} \leq m$

Now take $y \in X \backslash\{x\}$ and $\varepsilon>0$, and choose $f \in M_{[1]}$ such that $|f(y)-f(x)|>\left\|\varepsilon_{y}-\varepsilon_{x}\right\|-\varepsilon$. Then, using equation (2.1), we have

$$
\left\|\varepsilon_{y}-\varepsilon_{x}\right\|-\varepsilon \leq m\left\|\varepsilon_{y}-\varepsilon_{x}\right\|^{2}
$$

and so $\left\|\varepsilon_{y}-\varepsilon_{x}\right\| \geq 1 / m$. This shows that $x$ is an isolated point with respect to the Gleason metric on $X$.

We now give a theorem about members of class $\mathcal{C}_{X}$, where $X$ is a nonempty, compact set in $\mathbb{C}$, as defined on page 5 ; the theorem shows that properties (I) - (VI) are mutually equivalent for maximal ideals in these algebras. We shall see in Example 2.7 that these properties are not necessarily equivalent to property (VII) for algebras in the class $\mathcal{C}_{X}$.

Theorem 2.4. Let $X$ be a non-empty, compact plane set with $|X| \geq 2$, and let $A$ be a uniform algebra in the class $\mathcal{C}_{X}$. Then the following conditions on $z \in X$ are equivalent:
(a) z is a peak point for $A$;
(b) $M_{z}$ has a bounded approximate identity;
(c) $\{z\}$ is a one-point Gleason part;
(d) $M_{z}$ has a contractive pointwise approximate identity;
(e) $z$ is isolated with respect to the Gleason metric;
(f) null sequences in $M_{z}$ factor;
(g) $M_{z}$ factors;
(h) $M_{z}$ factors weakly;
(i) $M_{z}$ factors projectively.

Proof. The equivalence of (a) and (b) was noted in Proposition 1.3 for general uniform algebras on metrizable spaces, and the equivalence of (c) and (d) is Proposition 2.1. The implications (a) $\Rightarrow(\mathrm{c}) \Rightarrow(\mathrm{e})$ and (f) $\Rightarrow(\mathrm{g}) \Rightarrow$ $(\mathrm{h}) \Rightarrow$ (i) are trivial. The implications (b) $\Rightarrow(\mathrm{f})$ and (i) $\Rightarrow$ (e) are Theorem 1.5 and Proposition 2.3, respectively.

As noted by O'Farrell [27, p. 408], the metric density theorem of Browder [3, Theorem 3.3.9] (see also [33, Theorem 26.12]) applies to each algebra $A$ in the class $\mathcal{C}_{X}$, and so this implies that (e) $\Rightarrow(\mathrm{a})$.

Thus all the conditions (a) - (i) are equivalent. 
Geometric conditions on the set $X$ of the above theorem that show when a point $z \in X$ is a peak point with respect to the uniform algebra $A$ are given in [27].

We shall consider the big disc algebra. To define this algebra, take an irrational number $\alpha$ with $0<\alpha<1$, and consider the 'open half-plane' $H_{\alpha}$ consisting of the points $(m, n) \in \mathbb{Z} \times \mathbb{Z}$ with $m+n \alpha>0$. Note that $H_{\alpha}$ is a sub-semigroup of $\mathbb{Z} \times \mathbb{Z}$. Then consider monomials on $\mathbb{C}^{2}$ of the form $Z^{m} W^{n}$, where $(m, n) \in H_{\alpha}$; here $Z$ and $W$ are the coordinate functionals on $\mathbb{C}^{2}$. We take $\mathfrak{M}_{0, \alpha}$ to be the linear span of these monomials, and $\mathfrak{M}_{\alpha}$ and $\mathfrak{A}_{\alpha}$ to be the uniform closures of $\mathfrak{M}_{0, \alpha}$ and $\mathfrak{M}_{0, \alpha}^{\sharp}$, respectively, regarded as subalgebras of $C\left(\mathbb{T}^{2}\right)$. Then $\mathfrak{A}_{\alpha}$ is a uniform algebra on its character space $\Phi_{\alpha}$ that can be identified with the space $\mathbb{T}^{2} \times[0,1]$, with the subset $\mathbb{T}^{2} \times\{0\}$ identified to a point, called $x_{0}$; the corresponding maximal ideal in $\mathfrak{A}_{\alpha}$ at $x_{0}$ is $\mathfrak{M}_{\alpha}$. The set $\left\{x_{0}\right\}$ is a one-point Gleason part, but $x_{0} \notin \Gamma\left(\mathfrak{A}_{\alpha}\right)$ and, in particular, $\mathfrak{M}_{\alpha}$ does not have a bounded approximate identity. See [33, $\S 18]$ and the discussion in [19].

We shall now show that $\mathfrak{M}_{\alpha}$ factors projectively, but we do not know whether it factors or factors weakly, or whether null sequences in $\mathfrak{M}_{\alpha}$ factor.

Proposition 2.5. The maximal ideal $\mathfrak{M}_{\alpha}$ of the big disc algebra factors projectively, and so $\mathfrak{M}_{\alpha}$ satisfies (VI), but not (I).

Proof. We shall apply Proposition 1.15, taking $S$ to be the set $\mathfrak{M}_{0, \alpha}$, which is dense in $\mathfrak{M}_{\alpha}$.

Indeed, take $f=\sum_{j=1}^{n} \alpha_{j} Z^{m_{j}} W^{n_{j}}$ in $S$, where $n \in \mathbb{N},\left(m_{j}, n_{j}\right) \in H_{\alpha}$ for $j \in \mathbb{N}_{n}$, and $\alpha_{1}, \ldots, \alpha_{n} \in \mathbb{C}$, and set

$$
\varepsilon=\min \left\{m_{j}+n_{j} \alpha: j \in \mathbb{N}_{n}\right\},
$$

so that $\varepsilon>0$. It follows from Dirichlet's theorem on Diophantine approximation that there exist $p, q \in \mathbb{N}$ (with $q>2 / \varepsilon$ ) such that

$$
\alpha-\frac{\varepsilon}{q}<\frac{p}{q}<\alpha
$$

Take $j \in \mathbb{N}_{n}$, and consider the two points $(-p, q)$ and $\left(p+m_{j},-q+n_{j}\right)$. It follows from inequality (2.2) that both these points are in $H_{\alpha}$, and clearly their sum is $\left(m_{j}, n_{j}\right)$. Thus the elements $Z^{m_{j}} W^{n_{j}}$ of $\mathfrak{M}$ have as a common factor $Z^{-p} W^{q}$, and so there exists a function $g \in S$ with $f=Z^{-p} W^{q} g$. Clearly $\left|Z^{-p} W^{q}\right|_{\mathbb{T} \times \mathbb{T}}=1$ and $|f|_{\mathbb{T} \times \mathbb{T}}=|g|_{\mathbb{T} \times \mathbb{T}}$. Thus it follows from Proposition 1.15 that the maximal ideal $\mathfrak{M}_{\alpha}$ factors projectively. 
Example 2.6. In [8, Theorem 2.3], there is a construction of a separable uniform algebra on a non-empty, compact space $X$ such that $\left\{f^{2}: f \in A\right\}$ is dense in $A$, but such that $\Gamma(A) \subsetneq X$. Take $x \in X$. It follows easily that $\left\{f^{2}: f \in M_{x}\right\}$ is dense in $M_{x}$, and so, by Proposition 2.2, $\{x\}$ is a onepoint Gleason part. By Corollary 1.17, $M_{x}$ factors projectively. Thus every maximal ideal in $A$ factors projectively, but $M_{x}$ does not have a bounded approximate identity when $x \in X \backslash \Gamma(A)$. Again, for each $x \in X \backslash \Gamma(A)$, we do not know whether $M_{x}$ satisfies any of the conditions (II)-(V).

Example 2.7. Let $X$ be the compact plane set specified in Wermer's example that is given in [35]. For this example, $R(X) \neq C(X)$, and so, by [33, Theorem 26.8], there are points $x \in X$ that are not peak points for $R(X)$. By Theorem 2.4, $M_{x}$ does not factor projectively. However, each maximal ideal in $R(X)$ factors densely because, for this example, there are no nonzero, continuous point derivations at any point of $X$, and this shows that $(\mathrm{VII}) \not \Rightarrow(\mathrm{VI})$ in the class of maximal ideals of separable uniform algebras.

For another example, consider the 'road-runner' set, defined as follows [16, p. 52]. Let $X$ be the compact space in $\mathbb{C}$ obtained by deleting from $\overline{\mathbb{D}}$ a sequence $\left(D_{n}=\mathbb{D}\left(x_{n}, r_{n}\right): n \in \mathbb{N}\right)$ of open discs, where we ensure that the closed discs $\overline{D_{n}}$ are pairwise-disjoint and that the sequence $\left(x_{n}\right)$ in $\mathbb{R}^{+} \bullet$ decreases to 0 . Consider the maximal ideal

$$
M_{0}=\{f \in R(X): f(0)=0\} .
$$

It follows from Melnikov's Criterion [16, VIII, Theorem 4.5] that $M_{0}$ satisfies the equivalent properties (I) - (VI) if and only if $\sum_{n=1}^{\infty} r_{n} / x_{n}=\infty$, and it follows from a result of Hallstrom [20, p. 156] that $M_{0}$ satisfies property (VII) if and only if the strictly weaker condition that $\sum_{n=1}^{\infty} r_{n} / x_{n}^{2}=\infty$ holds. Thus again we see that (VII) $\nRightarrow$ (VI) in the class of maximal ideals of separable uniform algebras.

Finally, we note that Sidney in [34] constructed a natural, separable uniform algebra on a compact space $X$ and a point $x \in X$ such that $\{x\}$ is a one-point Gleason part, but such that $M_{x}$ does not factor densely. In this example, $x \notin \Gamma(A)$. In [18], it is shown that there is a natural, separable uniform algebra on a compact space $X$ and $x \in X$ such that each point of $X$ is a one-point part, but $M_{x}$ does not factor densely. In [5, Theorem 1.1], there is a triply-generated uniform algebra $A$ with a proper Šilov boundary, 
but where every point of $\Phi_{A}$ is a one-point part, and there are no nonzero, continuous point derivations, so that every maximal ideal in $A$ factors densely.

\section{EXTENSIONS OF UNIFORM ALGEBRAS}

3.1. Introduction. For a main example, we first discuss a construction that shares some properties with one originally due to Cole [4]; an exposition of Cole's construction is given in [33, §19]. Indeed, we shall describe certain classes of extensions of uniform algebras that include many standard extensions that have been discussed in the literature; in particular, such classes are discussed in [15, Lemmas 2.12 and 2.13 and §5]. For some similar classes of extensions of uniform algebras, see the paper [26] of Morley.

Let $X$ and $Y$ be compact spaces, and suppose that $\Pi: Y \rightarrow X$ is a continuous surjection. For $x \in X$, we define the fibre above $x$ to be

$$
F_{x}=\{y \in Y: \Pi(y)=x\} .
$$

The map $\Pi^{*}: C(X) \rightarrow C(Y)$ is defined by the formula

$$
\Pi^{*}(f)=f \circ \Pi \quad(f \in C(X)),
$$

so that $\Pi^{*}$ is an isometric isomorphism of $C(X)$ onto a closed subalgebra of $C(Y)$. A linear contraction $T: C(Y) \rightarrow C(X)$ such that $T \circ \Pi^{*}=I_{C(X)}$ is an averaging operator for $\Pi$; see [7, Definition 3.2.5].

For a subset $S$ of $\mathbb{C}$, we denote the closure of the convex hull of $S$ by $\overline{\mathrm{co}} S$.

Definition 3.1. Let $X$ be a compact space, take $x_{0} \in X$, and let $A$ be a uniform algebra on $X$. Then $\left(X, x_{0}, A\right)$ is a distinguished-point uniform algebra. Now suppose that $\left(Y, y_{0}, B\right)$ is also a distinguished-point uniform algebra. Then $\left(Y, y_{0}, B\right)$ is a distinguished-point extension of $\left(X, x_{0}, A\right)$ with respect to a continuous surjection $\Pi: Y \rightarrow X$ and an averaging operator $T: C(Y) \rightarrow C(X)$ for $\Pi$ if the following conditions are satisfied:

(i) $\Pi^{*}(A) \subset B$;

(ii) $F_{x_{0}}=\left\{y_{0}\right\}$;

(iii) $T(B)=A$;

(iv) $(T h)(x) \in \overline{\mathrm{co}} h\left(F_{x}\right)(x \in X, h \in C(Y))$.

In fact, clause (iv), above, follows from the other conditions on $T$ : see, for example, [26, Lemma 2.7]. Further immediate consequences of clauses 
(ii) and (iv) are the following additional properties of $T$, which we shall use below:

(v) $(T h)\left(x_{0}\right)=h\left(y_{0}\right) \quad(h \in C(Y))$;

(vi) $|(T h)(x)| \leq|h|_{F_{x}}(x \in X, h \in C(Y))$.

Proposition 3.2. Let $\left(X, x_{0}, A\right)$ be a distinguished-point uniform algebra, and suppose that $\left(Y, y_{0}, B\right)$ is a distinguished-point extension of $\left(X, x_{0}, A\right)$. Then $x_{0}$ is a strong boundary point for $A$ if and only if $y_{0}$ is a strong boundary point for $B$.

Proof. It is immediate that $y_{0}$ is a strong boundary point with respect to $B$ whenever $x_{0}$ is a strong boundary point with respect to $A$.

Now suppose that $y_{0}$ is a strong boundary point with respect to $B$, and take a neighbourhood $U$ of $x_{0}$ in $X$. Set $W=\Pi^{-1}(U)$, a neighbourhood of $y_{0}$ in $Y$. Then there exists $h \in B$ such that $h\left(y_{0}\right)=|h|_{Y}=1$ and $|h|_{Y \backslash W}<1$. But then $(T h)\left(x_{0}\right)=|T h|_{X}=1$, and it follows from clause (vi), above, that $|T h|_{X \backslash U}<1$. Thus $x_{0}$ is a strong boundary point with respect to $A$.

We shall also need the notion of a system of distinguished-point extensions. See [15, §5] for earlier examples of such systems.

Definition 3.3. Let $\left(X_{0}, x_{0}, A_{0}\right)$ be a distinguished-point uniform algebra, and take $\kappa$ to be an ordinal. A system

$$
\left(\left(X_{\alpha}, x_{\alpha}, A_{\alpha}\right) ; \Pi_{\alpha, \beta} ; T_{\alpha, \beta}: 0 \leq \alpha \leq \beta \leq \kappa\right)
$$

is a compatible system of distinguished-point extensions if

$$
\left(X_{\alpha}, \Pi_{\alpha, \beta}: 0 \leq \alpha \leq \beta \leq \kappa\right)
$$

is a projective system of non-empty, compact spaces and continuous surjections, if each $\left(X_{\alpha}, x_{\alpha}, A_{\alpha}\right)$ for $0 \leq \alpha \leq \kappa$ is a distinguished-point uniform algebra, and if, further:

(i) the maps $\Pi_{\alpha, \alpha}$ and $T_{\alpha, \alpha}$ are the identity maps on $X_{\alpha}$ and $C\left(X_{\alpha}\right)$, respectively, for each $0 \leq \alpha \leq \kappa$;

(ii) $\left(X_{\beta}, y_{\beta}, A_{\beta}\right)$ is a distinguished-point extension of $\left(X_{\alpha}, y_{\alpha}, A_{\alpha}\right)$ with respect to the maps $\Pi_{\alpha, \beta}: X_{\beta} \rightarrow X_{\alpha}$ and $T_{\alpha, \beta}: C\left(X_{\beta}\right) \rightarrow C\left(X_{\alpha}\right)$ whenever $0 \leq \alpha<\beta \leq \kappa$

(iii) $T_{\alpha, \gamma}=T_{\alpha, \beta} \circ T_{\beta, \gamma}$ whenever $0 \leq \alpha \leq \beta \leq \gamma \leq \kappa$;

(iv) for each limit ordinal $\gamma$ with $\gamma \leq \kappa$, the system

$$
\left(X_{\alpha}, \Pi_{\alpha, \beta}: 0 \leq \alpha \leq \beta \leq \gamma\right)
$$


is a directed system, the space $X_{\gamma}$ is the inverse limit of this system, and $\Pi_{\alpha, \gamma}$ is the canonical projection of $X_{\gamma}$ onto $X_{\alpha}$ for $0 \leq \alpha<\gamma$, and, further, the space

$$
\bigcup\left\{\Pi_{\alpha, \gamma}^{*}\left(A_{\alpha}\right): 0 \leq \alpha<\gamma\right\}
$$

is dense in $A_{\gamma}$.

Systems of distinguished-point extensions can be constructed by transfinite induction in a similar way to standard systems of Cole extensions, as described in [33, §19], for example. We shall sketch one such construction in $\S 3.2$ when proving Theorem 3.4. The key is to explain how to choose $\left(X_{\alpha+1}, y_{\alpha+1}, A_{\alpha+1}\right)$ to be a suitable distinguished-point extension of $\left(X_{\alpha}, y_{\alpha}, A_{\alpha}\right)$ for the relevant ordinal numbers $\alpha$, and then the compatibility conditions given above are essentially sufficient to conclude the proof; this parallels the usual theory of systems of Cole extensions as given in $[4,15,33]$, and elsewhere.

3.2. Proof of Theorem 1.6. We are seeking a maximal ideal $M$ in a uniform algebra $A$ such that null sequences in $M$ factor, but $M$ does not have a bounded approximate identity. In fact, we shall prove a rather stronger theorem, as follows.

Theorem 3.4. There are a natural uniform algebra $A$ on a compact space $X$ and a point $x \in X$ such that $M_{x}$ is non-zero and null sequences in $M_{x}$ factor, but such that $M_{x}$ does not have a bounded approximate identity, equivalently, $x$ is not a strong boundary point for $A$, and, further, such that each element in $M_{x}$ is the square of another element in $M_{x}$ and $\{y\}$ is a one-point Gleason part with respect to A for each $y \in X$.

As a first step, we shall show how, given a maximal ideal $M$ in a uniform algebra $A$, there is a (much) bigger uniform algebra in which (a copy of) each null sequence in $M$ factors.

Theorem 3.5. Let $A$ be a uniform algebra on a compact space $X$, and take $x_{0} \in X$. Then there are a uniform algebra $B$ on a compact space $Y$, a point $y_{0}$ in $Y$, a continuous surjection $\Pi: Y \rightarrow X$, and a linear contraction $T: C(Y) \rightarrow C(X)$ with the following properties:

(i) $\left(Y, y_{0}, B\right)$ is a distinguished-point extension of $\left(X, x_{0}, A\right)$ with respect to $\Pi$ and $T$;

(ii) for each null sequence $\left(f_{n}\right)$ in $M_{x_{0}}$, the null sequence $\left(\Pi^{*}\left(f_{n}\right)\right)$ factors in $M_{y_{0}}$; 
(iii) $y_{0}$ is a strong boundary point for $B$ if and only if $x_{0}$ is a strong boundary point for $A$.

Proof. To avoid notational complexity, we shall show how, given one fixed null sequence, say $\left(f_{n}\right)$, in $M_{x_{0}}$, we can obtain a uniform algebra $B$ on a compact space $Y, y_{0} \in Y$, a continuous surjection $\Pi: Y \rightarrow X$, and a linear contraction $T: C(Y) \rightarrow C(X)$ such that the particular null sequence $\left(\Pi^{*}\left(f_{n}\right)\right)$ factors in $M_{y_{0}}$ and the other clauses hold; the more general result follows by a small modification of the following argument.

We may suppose that $\left|f_{n}\right|_{X} \leq 1(n \in \mathbb{N})$. For $x \in X$, set

$$
k_{x}:=\max \left\{\left|f_{n}(x)\right|^{1 / 2}: n \in \mathbb{N}\right\}
$$

Note that the constant $k_{x}$ is well-defined because $\left|f_{n}(x)\right| \rightarrow 0$ as $n \rightarrow \infty$ for each $x \in X$. Moreover, since $\left(f_{n}\right)$ is a null sequence, it is easy to see that the map $x \mapsto k_{x}, X \rightarrow \mathbb{R}^{+}$, is continuous.

The space $Y$ is defined to be a subspace of the space $X \times \mathbb{C}^{\mathbb{N}} \times \mathbb{C}$ that satisfies certain conditions. To define these conditions, we take a generic point of $X \times \mathbb{C}^{\mathbb{N}} \times \mathbb{C}$ to have the form $\left(x,\left(z_{n}\right), w\right)$, where $x \in X$, where $z_{n} \in \mathbb{C}(n \in \mathbb{N})$, and where $w \in \mathbb{C}$. The conditions on each point $\left(x,\left(z_{n}\right), w\right)$ are the following:

(i) $z_{n} w=f_{n}(x)(n \in \mathbb{N})$;

(ii) $|w|=k_{x}$;

(iii) $\left|z_{n}\right|^{2} \leq\left|f_{n}(x)\right| \quad(n \in \mathbb{N})$.

For each such point $\left(x,\left(z_{n}\right), w\right)$, we see that $\left|z_{n}\right| \leq 1 \quad(n \in \mathbb{N})$ and that $|w| \leq 1$, and so $Y$ is contained in the compact space $X \times \overline{\mathbb{D}}^{\mathbb{N}} \times \overline{\mathbb{D}}$. Further, $Y$ is closed in this space, and so $Y$ is a compact space. Take $n \in \mathbb{N}$. Then it follows from (ii) and (iii) that $\left|z_{n}\right| \leq|w|$; if $f_{n}(x)=0$, then $z_{n}=0$.

We define

$$
\Pi:\left(x,\left(z_{n}\right), w\right) \mapsto x, \quad Y \rightarrow X
$$

so that $\Pi$ is a continuous surjection.

We also define

$$
p_{n}:\left(x,\left(z_{n}\right), w\right) \mapsto z_{n}, \quad Y \rightarrow \mathbb{C} \quad(n \in \mathbb{N}),
$$

and $q:\left(x,\left(z_{n}\right), w\right) \mapsto w, Y \rightarrow \mathbb{C}$, so that each map $p_{n}$ and the map $q$ belongs to $C(Y)$. Clearly

$$
p_{n} q=\Pi^{*}\left(f_{n}\right) \quad(n \in \mathbb{N}) .
$$


The algebra $B$ is defined to be the smallest closed subalgebra of $C(Y)$ containing $\Pi^{*}(A)$ and all of the functions $p_{n}$ and $q$. Since $B$ separates the points of $Y$, the algebra $B$ is a uniform algebra on $Y$.

There is an obvious jointly-continuous action of the circle group $\mathbb{T}$ on $Y$ defined by

$$
\left(\zeta,\left(x,\left(z_{n}\right), w\right)\right) \mapsto\left(x,\left(z_{n} / \zeta\right), w \zeta\right) \quad\left(\zeta \in \mathbb{T},\left(x,\left(z_{n}\right), w\right) \in Y\right)
$$

This action will help us to define a suitable averaging map below.

For $x \in X$, we again define the fibre $F_{x}=\Pi^{-1}(\{x\})$.

First, suppose that $k_{x}=0$. Then $F_{x}$ is the single point $(x, 0,0)$. In particular, $k_{x_{0}}=0$, and so $F_{x_{0}}$ is a singleton in $Y$, say $F_{x_{0}}=\left\{y_{0}\right\}$.

Second, suppose that $k_{x}>0$. Then an element $\left(x,\left(z_{n}\right), w\right) \in Y$ is such that $w \neq 0$, and $F_{x}$ is the circle

$$
F_{x}=\left\{\left(x,\left(f_{n}(x) / k_{x}\right) \mathrm{e}^{-\mathrm{i} \theta}, k_{x} \mathrm{e}^{\mathrm{i} \theta}\right): 0 \leq \theta<2 \pi\right\} .
$$

Clearly $p_{n} \in M_{y_{0}}$ and $\left|p_{n}\right|_{Y}^{2} \leq\left|f_{n}\right|_{X}$ for each $n \in \mathbb{N}$, and so $\left(p_{n}\right)$ is a null sequence in $M_{y_{0}}$. Further, $q \in M_{y_{0}}$, and so equation (3.2) gives the required factorization of $\left(\Pi^{*}\left(f_{n}\right)\right)$ in $M_{y_{0}}$, thus establishing clause (ii) of the theorem.

We define a map $T: C(Y) \rightarrow \mathbb{C}^{X}$ as follows. Take $h \in C(Y)$ and $x \in X$. Suppose that $k_{x}=0$. Then $(T h)(x)=h(x, 0,0)$. Suppose that $k_{x}>0$. Then

$$
(T h)(x)=\frac{1}{2 \pi} \int_{0}^{2 \pi} h\left(x,\left(f_{n}(x) / k_{x}\right) \mathrm{e}^{-\mathrm{i} \theta}, k_{x} \mathrm{e}^{\mathrm{i} \theta}\right) \mathrm{d} \theta .
$$

In either case, $(T h)(x)$ is the average of the values in $h\left(F_{x}\right)$ obtained by using the Haar measure on $\mathbb{T}$ and the transitive action of $\mathbb{T}$ on the fibre $F_{x}$. Because the action of the compact group $\mathbb{T}$ on $Y$ is jointly continuous, it is not hard to see that $T h \in C(X)$ for each $h \in C(Y)$ : this is a standard argument, given, for example, in [4, Theorem 1.3], [33, Theorem 19.1(a)], and [26, Lemma 5.10]. It is now clear that the map $T: C(Y) \rightarrow C(X)$ is a linear contraction, and also that $T\left(\Pi^{*}(f)\right)=f(f \in C(X))$, and so $T \circ \Pi^{*}=I_{C(X)}$.

We next show that $T(B)=A$. Clearly we have $A=T\left(\Pi^{*}(A)\right) \subset T(B)$.

To prove that $T(B) \subset A$, first consider an element $h \in C(Y)$ of the form

$$
h=\Pi^{*}(f) p_{1}^{n_{1}} \cdots p_{k}^{n_{k}} q^{m},
$$

where $f \in A$ and $n_{1}, \ldots, n_{k}, m \in \mathbb{Z}^{+}$. We claim that $T h \in A$.

First suppose that $n_{1}+\cdots+n_{k}=m$. Then it is immediate that $T h \in A$. Second, suppose that $n_{1}+\cdots+n_{k} \neq m$. If $k_{x}=0$, then $(T h)(x)=0$, and, if $k_{x} \neq 0$, then $(T h)(x)$ is given by

$$
(T h)(x)=g(x) \int_{0}^{2 \pi} \exp \left(\mathrm{i}\left(m-n_{1}-\cdots-n_{k}\right) \theta\right) \mathrm{d} \theta
$$


for some $g \in C(X)$, and so again $(T h)(x)=0$. Thus $T h=0$, giving the claim in this case

It follows that $T h \in A$ whenever $h$ is a linear combination of functions specified in equation (3.4); this set of linear combinations is dense in $B$ and $T$ is continuous, and so $T h \in A$ for each $h \in B$. Thus $T(B) \subset A$. We have shown that $\left(Y, y_{0}, B\right)$ is a distinguished-point extension of $\left(X, x_{0}, A\right)$ with respect to $\Pi$ and $T$, thus giving clause (i) of the theorem.

Clause (iii) of the theorem follows from Proposition 3.2.

This completes the proof.

Remark. In the case where all Jensen measures (see [16, p. 33]) for $A$ are trivial, the same is true for $B$, and so $B$ is natural. For this, we first note that $B \mid F_{x}$ is dense in $\left(C\left(F_{x}\right),|\cdot|_{F_{x}}\right)$ for each $x \in X$, and then apply [26, Theorem 3.6].

In this setting, where $A$ and $B$ are natural, suppose that $\left\{x_{0}\right\}$ is a one-point part for $A$, and consider $y \in Y \backslash\left\{y_{0}\right\}$. Now set $x=\Pi(y)$, so that $x \in X \backslash\left\{x_{0}\right\}$ and there exists a sequence $\left(f_{n}\right)$ in $\left(M_{x_{0}}\right)_{[1]}$ such that $\lim _{n \rightarrow \infty} f_{n}(x)=1$. But now $\left(\Pi^{*}\left(f_{n}\right)\right)$ is a sequence in $\left(M_{y_{0}}\right)[1]$ such that $\lim _{n \rightarrow \infty} \Pi^{*}\left(f_{n}\right)(y)=1$, and so $y$ is in a different part to $y_{0}$. Hence $\left\{y_{0}\right\}$ is a one-point part for $B$.

The following result is standard; it is an extension of the constructions expounded in [33, §19] and is essentially [15, Lemma 2.5].

Theorem 3.6. Let $A$ be a uniform algebra on a compact space $X$, and take $x_{0} \in X$. Then there are a uniform algebra $B$ on a compact space $Y, a$ point $y_{0}$ in $Y$, a continuous surjection $\Pi: Y \rightarrow X$, and a linear contraction $T: C(Y) \rightarrow C(X)$ with the following properties:

(i) $\left(Y, y_{0}, B\right)$ is a distinguished-point extension of $\left(X, x_{0}, A\right)$ with respect to $\Pi$ and $T$;

(ii) for each $f \in M_{x_{0}}$, there exists $g \in M_{y_{0}}$ such that $g^{2}=\Pi^{*}(f)$;

(iii) $y_{0}$ is a strong boundary point for $B$ if and only if $x_{0}$ is a strong boundary point for $A$.

Proof of Theorem 3.4 We start with a (natural) uniform algebra $A_{0}$ on a compact space $X_{0}$ and a point $x_{0} \in X_{0}$ that is not a strong boundary point for $A_{0}$. For example, we can take $A_{0}$ to be the disc algebra and $x_{0}=0$. We shall construct a system

$$
\left(X_{\alpha}, x_{\alpha}, A_{\alpha}, \Pi_{\alpha, \beta}: 0 \leq \alpha \leq \beta \leq \omega_{1}\right\},
$$

consisting of successive distinguished-point extensions of $\left(X_{0}, x_{0}, A_{0}\right)$. 
Take $\alpha$ with $0 \leq \alpha<\omega_{1}$, and assume first that the system has been constructed to level $\alpha$. Set $\beta=\alpha+1$. In the case where $\alpha$ is a non-limit ordinal of the form $\gamma+n$, where $\gamma$ is a limit ordinal and $n \in \mathbb{Z}^{+}$is an odd number, the extension $\left(X_{\beta}, x_{\beta}, A_{\beta}\right)$ is formed from $\left(X_{\alpha}, x_{\alpha}, A_{\alpha}\right)$ by using Theorem 3.5. In the similar case where $n \in \mathbb{Z}^{+}$is an even number, the extension $\left(X_{\beta}, x_{\beta}, A_{\beta}\right)$ is formed from $\left(X_{\alpha}, x_{\alpha}, A_{\alpha}\right)$ by using the standard 'addition of square roots', as described in Theorem 3.6. Second, the case where $\alpha$ is a non-zero limit ordinal and we assume that the system has been constructed to level $\gamma$ for each $\gamma<\alpha$, the extension $\left(X_{\alpha}, x_{\alpha}, A_{\alpha}\right)$ is formed by taking a direct limit, as described above.

The resulting extension $\left(X_{\omega_{1}}, x_{\omega_{1}}, A_{\omega_{1}}\right)$ has all the required properties of the theorem, where we take $X$ to be the character space of the uniform algebra $A=A_{\omega_{1}}$; we note that $\{x\}$ is a one-point part for $A$ for each $x \in X$ by Proposition 2.2 .

Remark. As indicated in the previous remark, we could require that all our uniform algebras $A_{\alpha}$ have unique Jensen measures; in this case, each algebra $A_{\alpha}$, including $A$, is already natural.

We note again that our example is enormous, and certainly non-separable; we do not know how to modify the construction to exhibit a separable example.

\section{LOCAL FACTORIZATION}

4.1. Properties (A) and (B). We now introduce two further 'factorization-type' properties related to property (IV); they are 'local' in that they refer to the existence of elements with certain properties in an algebra.

Let $A$ be an algebra (not necessarily commutative), and let $E$ be a left $A$-module. Then

$$
A \cdot E=\{a \cdot x: a \in A, x \in E\} .
$$

Also, given a sequence $\left(a_{n}\right)$ in $A$, we define

$$
\lim _{\longleftarrow} a_{1} \cdots a_{n} \cdot E=\left\{\begin{array}{ll}
x \in E: & \text { there exists }\left(x_{n}\right) \text { in } E \text { such that } \\
& x=x_{1} \text { and } x_{n}=a_{n} \cdot x_{n+1}(n \in \mathbb{N})
\end{array}\right\},
$$

essentially as in $\S 1.1$. With this notation, we specify two properties (A) and (B):

(A) $\underset{\varliminf}{\lim } a^{n} \cdot A \neq\{0\}$ for some $a \in A$;

(B) $\lim _{\longleftarrow} a_{1} \cdots a_{n} \cdot A \neq\{0\}$ for some sequence $\left(a_{n}\right)$ in $A$. 
The two conditions (A) and (B) in the case of commutative, radical Banach algebras are parts of a classification scheme due to Esterle [14]; this classification scheme is expounded in [6, §4.9], and properties (A) and (B) correspond to classes (III) and (IV) in that classification. It is left open in $[6,14]$ whether or not the two classes (III) and (IV) of Esterle are distinct (and we do not resolve this point).

Clearly $(\mathrm{A}) \Rightarrow(\mathrm{B})$ and $(\mathrm{IV}) \Rightarrow(\mathrm{B})$. In fact, Corollary 5.4, below, will show that a weaker condition than (IV) is sufficient to imply (B).

Proposition 4.1. Let $A$ be a non-zero, commutative Banach algebra satisfying property (II). Then A also satisfies property (A).

Proof. Note that $A$ factors. Take any element $a \in A$ with $a \neq 0$, and then take $b_{1}, c_{1} \in A$ with $a=b_{1} c_{1}$.

Now we inductively define two sequences $\left(b_{n}\right)$ and $\left(c_{n}\right)$ in $A$ such that $c_{n}=b_{n+1} c_{n+1}$; by scaling, we may suppose that $\left\|b_{n}\right\| \leq 1 / n$ for each $n \in \mathbb{N}$, and so $\left(b_{n}\right)$ is a null sequence. By (II), there exist $b \in A^{\bullet}$ and a null sequence $\left(d_{n}\right)$ in $A$ such that $b_{n}=b d_{n}(n \in \mathbb{N})$. Set $a_{n}=c_{n} d_{1} \cdots d_{n}(n \in \mathbb{N})$. Then $a_{n}=b a_{n+1}(n \in \mathbb{N})$, and so $a \in \lim ^{\lim } b^{n} \neq\{0\}$, as required.

Example 4.2. Let $X$ be a compact plane set containing the point 0, and take $M$ to be the maximal ideal at 0 in $R(X)$, and suppose that $M^{2}$ is dense in $M$.

We claim that $M=\overline{Z M}$, where $Z$ is the coordinate functional. To see this, take $f \in M$ and $\varepsilon>0$. Then there exists $q \in M^{2}$ with $|f-q|_{X}<\varepsilon$, say $q=g_{1} h_{1}+\cdots+g_{k} h_{k}$, where $g_{j}, h_{j} \in M\left(j \in \mathbb{N}_{k}\right)$. Since the rational functions with poles off $X$ are uniformly dense in $R(X)$, those that vanish at 0 are uniformly dense in $M$. Thus, for each $j \in \mathbb{N}_{k}$, the function $g_{j}$ can be approximated arbitrarily well by a function of the form $Z f_{j}$, where $f_{j}$ is a rational function with poles off $X$. In particular, there exist functions $f_{1}, \ldots, f_{k} \in R(X)$ such that

$$
\left|f-\sum_{j=1}^{k} Z f_{j} h_{j}\right|_{X}<\varepsilon .
$$

But $\sum_{j=1}^{k} f_{j} h_{j} \in M$, and so $M=\overline{Z M}$, as claimed.

It again follows from the Mittag-Leffler theorem, Theorem 1.1, that $M$ satisfies (A), with the element $b$ taken to be $Z$, and hence $M$ also satisfies (B).

In particular, take $X$ to be a road-runner set as described in Example 2.7; we noted that there are examples of such sets such that $M$ satisfies (VII), 
but does not satisfy the equivalent conditions (I) - (VI). Thus condition (A) does not imply condition (VI) in the class of maximal ideals in uniform algebras of the form $R(X)$.

We do not know whether properties (III) or (IV) (for a commutative Banach algebra) implies property (A).

Note that the maximal ideal $M_{x}$ in $H^{\infty}(\mathbb{D})$ mentioned on page 11 , which satisfies property (IV), is not a counter-example to the implication that $(\mathrm{IV}) \Rightarrow(\mathrm{A})$. Indeed, set $M:=\{f \in A(\overline{\mathbb{D}}): f(1)=0\}$. Then $M$ has a bounded approximate identity, and so, by Proposition 4.1, $M$ satisfies (A). Since there is an isometric embedding of $M$ into $M_{x}$, it follows that $M_{x}$ also satisfies property (A).

4.2. An example. We have stated that we do not know an example of a commutative, radical Banach algebra that satisfies property (B), but does not satisfy property (A). However we can present an example of a maximal ideal in a separable uniform algebra that satisfies (B), but not (A).

We first give some further notation. For a complex number $z$, we set $x=\Re z$ and $y=\Im z$, so that $z=x+\mathrm{i} y$. The open half-plane $\Pi$ is defined by

$$
\Pi:=\{z \in \mathbb{C}: x>0\} .
$$

Throughout this subsection, we shall take

$$
A:=A^{b}(\bar{\Pi})
$$

to be the algebra of all bounded, continuous functions on $\bar{\Pi}$ that are analytic on $\Pi$, so that $A$ is a uniform algebra on its compact character space; recall $\left[6\right.$, A.2.25] that $|F|_{\Pi}$ is equal to $\sup \{|F(\mathrm{i} y)|: y \in \mathbb{R}\}$ for each $F \in A$.

We shall use the Ahlfors-Heins theorem [6, Theorem A.2.47] in the following form.

Theorem 4.3. Let $F \in A^{\bullet}$. Then there exists $c \geq 0$ such that

$$
\lim _{r \rightarrow \infty} \frac{1}{r} \log \left|F\left(r \mathrm{e}^{\mathrm{i} \theta}\right)\right|=-c \cos \theta \quad \text { for almost all } \quad \theta \in(-\pi / 2, \pi / 2) .
$$

Definition 4.4. For $a>0$, the set $I_{a}$ consists of functions $F \in A$ with $|F(z)|=O\left(\mathrm{e}^{-a x}\right)$ as $z \rightarrow \infty$ in $\bar{\Pi}$. Further, $I=\bigcup\left\{I_{a}: a>0\right\}$.

Clearly $I_{a}$ is an ideal in $A$ for each $a>0$, and $I$ is also an ideal in $A$.

Lemma 4.5. Suppose that $F \in I$. Then $\bigcap_{n=1}^{\infty} F^{n} A=\{0\}$. 
Proof. Take $a>0$ such that $F \in I_{a}$. Then $F^{n} \in I_{n a}$ for each $n \in \mathbb{N}$, and so, for each $G \in \bigcap_{n=1}^{\infty} F^{n} A$, necessarily $G \in I_{a}$ for each $a>0$.

Assume towards a contradiction that $G \neq 0$. Take $c \geq 0$ to be the constant specified in equation (4.1) (with respect to the function $G$ ), and take $\theta \in(-\pi / 2, \pi / 2)$ such that the the limit in (4.1) exists for this value of $\theta$. For each $a>0$, we have

$$
\limsup _{r \rightarrow \infty} \frac{1}{r} \log \left|G\left(r \mathrm{e}^{\mathrm{i} \theta}\right)\right| \leq-a \cos \theta,
$$

and so $c \geq a$ for each $a>0$, a contradiction. Hence $G=0$, giving the result.

The Banach space of all complex-valued, regular Borel measures on $\mathbb{R}^{+}$ is $M\left(\mathbb{R}^{+}\right)$, so that $M\left(\mathbb{R}^{+}\right)$is a commutative, unital, semi-simple Banach algebra with respect to the convolution product $\star$; see $[6, \S 4.7]$. The Laplace transform

$$
\mathcal{L}: \mu \rightarrow \mathcal{L} \mu, \quad\left(M\left(\mathbb{R}^{+}\right), \star\right) \rightarrow(A, \cdot),
$$

where $(\mathcal{L} \mu)(z)=\int_{\mathbb{R}^{+}} \mathrm{e}^{-z t} \mathrm{~d} \mu(t) \quad(z \in \bar{\Pi})$, is an injective linear contraction and an algebra homomorphism, and so we may regard $M\left(\mathbb{R}^{+}\right)$as a Banach function algebra on $\bar{\Pi}$. Define

$$
\alpha(\mu):=\inf \operatorname{supp} \mu \quad\left(\mu \in M\left(\mathbb{R}^{+}\right)^{\bullet}\right)
$$

as in [6, Definition 4.7.18]. Then Titchmarsh's convolution theorem [6, Theorem 4.7.22] shows that

$$
\alpha(\mu \star \nu)=\alpha(\mu)+\alpha(\nu) \quad\left(\mu, \nu \in M\left(\mathbb{R}^{+}\right)^{\bullet}\right) .
$$

Let $\mu \in M\left(\mathbb{R}^{+}\right)$. Then $\mathcal{L} \mu \in I_{a}$ if and only if $\alpha(\mu) \geq a[6$, Proposition 4.7.19].

Consider the semigroup algebra $\left(\ell^{1}\left(\mathbb{R}^{+}\right), \star\right)$, regarded as a closed subalgebra of the algebra $\left(M\left(\mathbb{R}^{+}\right), \star\right)$, and take an element

$$
f=\sum\left\{\alpha_{r} \delta_{r}: r \in \mathbb{R}^{+}\right\} \in \ell^{1}\left(\mathbb{R}^{+}\right)
$$

so that

$$
(\mathcal{L} f)(z)=\sum\left\{\alpha_{r} \mathrm{e}^{-z r}: r \in \mathbb{R}^{+}\right\} \quad(z \in \bar{\Pi})
$$

and $\ell^{1}\left(\mathbb{R}^{+}\right)$is a subalgebra of $A$. Set $E_{r}=\mathcal{L}\left(\delta_{r}\right)\left(r \in \mathbb{R}^{+}\right)$.

Definition 4.6. Denote by $M$ and $B$, respectively, the closures in $\left(A,|\cdot|_{\bar{\Pi}}\right)$ of the sets $\left\{\mathcal{L} f: f \in \ell^{1}\left(\mathbb{Q}^{+\bullet}\right)\right\}$ and $\left\{\mathcal{L} f: f \in \ell^{1}\left(\mathbb{Q}^{+}\right)\right\}$. 
Thus $B$ is a separable, unital, closed subalgebra of $A$, so that $B$ is a uniform algebra on its compact character space; $M$ is a maximal ideal in $B$, and $M \cap I$ is dense in $M$. In fact, $M$ is equal to $\operatorname{ker} \varphi$, where

$$
\varphi(F):=\lim _{x \rightarrow \infty} F(x) \quad(F \in B) .
$$

To see this, we write $\ell_{00}^{1}\left(\mathbb{Q}^{+\bullet}\right)$ for the dense subalgebra of $\ell^{1}\left(\mathbb{Q}^{+\bullet}\right)$ that is equal to $\operatorname{lin}\left\{\delta_{r}: r \in \mathbb{Q}^{+\bullet}\right\}$, and $L_{00}$ for the space of Laplace transforms of elements of $\ell_{00}^{1}\left(\mathbb{Q}^{+\bullet}\right)$. Take $F \in M$ and $\varepsilon>0$. Then there exists $H \in L_{00}$ with $|F-H|_{\bar{\Pi}}<\varepsilon$. Clearly, there exists $x_{0} \in \mathbb{R}^{+}$with $|H(x)|<\varepsilon\left(x \geq x_{0}\right)$, and this implies that $|F(x)|<2 \varepsilon\left(x \geq x_{0}\right)$. Thus $\lim _{x \rightarrow \infty} F(x)=0$, as required.

Note that the algebra that is the closure in $A$ of $\left\{\mathcal{L} f: f \in \ell^{1}\left(\mathbb{R}^{+}\right)\right\}$is not separable because $\left|E_{r}-E_{s}\right|_{\bar{\Pi}}=2$ for $r, s \in \mathbb{R}^{+}$with $r \neq s$. This implies that the Banach algebra $A$ is not separable.

Lemma 4.7. The maximal ideal $M$ in the uniform algebra $B$ factors projectively.

Proof. Consider the dense subset $S=\ell_{00}^{1}\left(\mathbb{Q}^{+\bullet}\right)$ of $\ell^{1}\left(\mathbb{Q}^{+\bullet}\right)$, and regard $S$ as a dense subset of $M$. Take $f \in S$, so that $f=g \star h$, where $g=\delta_{a}$ for some $a \in \mathbb{Q}^{+\bullet}$ and $h \in S$, as in Corollary 1.16. The Laplace transforms of $f, g$, and $h$ are $F, G$, and $H$, respectively, and clearly $|F|_{\bar{\Pi}}=|G|_{\bar{\Pi}}|H|_{\bar{\Pi}}$ because $|G(\mathrm{i} y)|=1(y \in \mathbb{R})$.

It again follows from Proposition 1.15 that $M$ factors projectively.

We now establish, in Theorem 4.9, a slight extension of a famous theorem of H. Bohr [6, Theorem 4.7.55].

Lemma 4.8. Let $G \in B$ be such that $G(z) \neq 0(z \in \Pi)$, and take $\tau>0$. Then

$$
\inf \{|G(\tau+\mathrm{i} y)|: y \in \mathbb{R}\}>0 .
$$

Proof. The argument of [6, Lemma 4.7.54] gives this result.

The following proof is close to that of [6, Theorem 4.7.55].

Theorem 4.9. Let $F \in M \backslash I$. Then there exists $z \in \Pi$ such that $F(z)=0$.

Proof. Assume toward a contradiction that $F(z) \neq 0(z \in \Pi)$. Take $\tau>0$, and apply the previous lemma to see that

$$
\inf \{|F(\tau+\mathrm{i} t)|: t \in \mathbb{R}\} \geq \eta
$$

for some $\eta>0$. Set $G(z):=F(z+\tau)$, so that $|G(\mathrm{i} t)|>\eta(t \in \mathbb{R})$. 
Since $G(z) \neq 0 \quad(z \in \Pi)$, it follows from Nevanlinna's theorem [6, A.2.46] that there exists $c \in \mathbb{R}$ such that

$$
\log |G(z)|=\frac{1}{\pi} \int_{-\infty}^{\infty} \frac{x}{x^{2}+(y-t)^{2}} \log |G(\mathrm{i} t)| \mathrm{d} t+c x \quad(z \in \Pi) .
$$

Take $a>0$. Since $G \notin I_{a}$, there is a sequence $\left(z_{k}=x_{k}+\mathrm{i} y_{k}\right)$ in $\Pi$ such that $x_{k} \rightarrow \infty$ as $k \rightarrow \infty$ and $\left|G\left(z_{k}\right)\right|>\mathrm{e}^{-a x_{k}}(k \in \mathbb{N})$. It follows from (4.2) that $-a \leq c \leq 0$. This holds for each $a>0$, and so $c=0$. It now follows from (4.2) that $|G(z)| \geq \eta(z \in \Pi)$. However $\lim _{x \rightarrow \infty} G(x)=0$ because $G \in M$, the required contradiction. Thus there exists $z \in \Pi$ such that $F(z)=0$.

Theorem 4.10. The maximal ideal $M$ in the separable uniform algebra $B$ factors projectively, and so satisfies (VI) and (B), but $M$ does not satisfy (A).

Proof. By Lemma 4.7, $M$ factors projectively, and so $M$ satisfies (B).

To show that the ideal $M$ does not satisfy (A), it suffices to show that $\bigcap F^{n} M=\{0\}$ for each $F \in M$. In the case where $F \in I$, this follows from Lemma 4.5. In the case where $F \in M \backslash I$, it follows from Theorem 4.9 that there exists $z \in \Pi$ such that $F(z)=0$. Thus each $G \in \bigcap F^{n} M$ is analytic on a neighbourhood of $z$ and has a zero of infinite order at $z$, and so $G=0$, giving the result in this case.

We can transfer the above algebras $B$ and $M$ from the half-plane $\Pi$ to the unit disc $\mathbb{D}$. Indeed, take $r \in \mathbb{R}^{+\bullet}$, and define

$$
f_{r}(z)=\exp \left(r\left(\frac{z+1}{z-1}\right)\right) \quad(z \in \overline{\mathbb{D}} \backslash\{1\}) .
$$

The function $f_{0}$ is the constant function 1 . Thus the functions $f_{r}$ for $r \in \mathbb{R}^{+}$ belong to $H^{\infty}(\mathbb{D})$. Clearly we can identify $B$ and $M$ with the closed subalgebras of the uniform algebra $H^{\infty}(\mathbb{D})$ that are generated by $\left\{f_{r}: r \in \mathbb{Q}^{+}\right\}$ and $\left\{f_{r}: r \in \mathbb{Q}^{+\bullet}\right\}$, respectively. Again denote by $\mathfrak{M}$ the character space of $H^{\infty}(\mathbb{D})$, as in [22, Chapter 10$]$ and [17, Chapter V, $\left.\S 1\right]$, so that $\mathbb{D}$ is dense in $\mathfrak{M}$ by Carleson's theorem [17, Chapter VIII]. Let $\mathfrak{M}_{1}$ be the fibre in $\mathfrak{M}$ that sits above the point 1 . The restriction of $H^{\infty}(\mathbb{D})$ (defined on $\mathfrak{M}$ ) to $\mathfrak{M}_{1}$ is called $A_{1}$ on page 187 of [22], and some properties of $A_{1}$ are given there. In particular, $A_{1}$ is a natural uniform algebra on $\mathfrak{M}_{1}$. The subset $Z$ of $\mathfrak{M}_{1}$ that is the common zero set of each of the functions $f_{r}$ for $r \in \mathbb{Q}^{+\bullet}$ is non-empty, is the union of Gleason parts for $H^{\infty}(\mathbb{D})$, and is disjoint from the Šilov boundary of $H^{\infty}(\mathbb{D})$. Consider the space that we shall call $K$ that is the quotient of $\mathfrak{M}_{1}$ formed by identifying the points of $\mathfrak{M}_{1}$ that are not 
separated by $B$. Then it can be seen that we can regard $B$ as a subalgebra of $A_{1}$, that $K$ is exactly the character space $\Phi_{B}$ of $B$, and that $B$ is a uniform algebra on $K$; the point $x_{0} \in K$ corresponding to the set $Z$ in $\mathfrak{M}_{1}$ corresponds to the maximal ideal $M$ in $B ;\left\{x_{0}\right\}$ is a one-point part off the Šilov boundary of $B$, and so $M$ does not have a bounded approximate identity. We do not know whether $M$ factors, but we have shown that it factors projectively.

\section{RADICAL ALGEBRAS}

5.1. Classification of radical algebras. The classification scheme of Esterle works in a more general setting than for commutative, radical Banach algebras, and we now explain this setting. We shall also expand this classification of Esterle somewhat, and give an application to a question of how many prime ideals such an algebra can possess.

In this section, an algebra $A$ is not necessarily commutative, unless this is stated.

Let $A$ be an algebra with a topology $\tau$, and suppose that $(A, \tau)$ is a (Hausdorff) topological linear space. Then $(A, \tau)$ is a topological algebra if the product map

$$
(a, b) \mapsto a b, \quad A \times A \rightarrow A,
$$

is continuous; a topological algebra is an (F)-algebra if there is a complete metric on $A$ that defines the topology $\tau$, and we then write $(A, d)$, where $d$ is a complete metric. Each Banach algebra is obviously an (F)-algebra.

The following lemma extends [14, Proposition 3.1]. Recall that an algebra $A$ acts continuously on a left $A$-module $E$ that is an $(\mathrm{F})$-space if the map $x \mapsto a \cdot x, E \rightarrow E$, is continuous for each $a \in A$.

Lemma 5.1. Let $A$ be an algebra, and let $E$ be a left $A$-module and an (F)-space with a complete metric $d$ such that $A$ acts continuously on $E$. Suppose that $S$ is a subset of $E$ such that $S \subset \overline{A \cdot S}$. Then, for each $x \in S$ and $\varepsilon>0$, there are a sequence $\left(a_{n}\right)$ in $A$ and $y \in \underset{\longleftarrow}{\lim } a_{1} \cdots a_{n} \cdot E$ with $d(x, y) \leq \varepsilon$.

Proof. For $n \in \mathbb{N}$, set $E_{n}:=A^{n-1} \times E$ equipped with the product metric (with $E_{1}=E$ ), where $A$ is given the discrete metric, and define a continuous $\operatorname{map} \theta_{n}: E_{n+1} \rightarrow E_{n}$ by

$$
\left(a_{1}, \ldots, a_{n}, x\right) \mapsto\left(a_{1}, \ldots, a_{n-1}, a_{n} x\right),
$$

so that $\left(\left(E_{n}, \theta_{n}\right): n \in \mathbb{N}\right)$ is a projective sequence. Set $X_{n}:=A^{n-1} \times S$. Then $X_{n} \subset \overline{\theta_{n}\left(X_{n+1}\right)}$, and so, by Theorem $1.1, X_{1}=S$ is contained in the 
closure of the set $\lim _{\longleftarrow} a_{1} \cdots a_{n} \cdot E$ for some sequence $\left(a_{n}\right)$ in $A$, which implies the lemma.

Lemma 5.2. Let $A$ be an algebra, and let $E$ be a left $A$-module and an (F)-space with a complete metric $d$. Suppose that $a \in A^{\bullet}$ is such that a acts continuously on $E$, and suppose that $S$ is a subset of $E$ with $S \subset \overline{a \cdot S}$. Then, for each $x \in S$ and $\varepsilon>0$, there exists $y \in \varliminf_{\longleftarrow} a^{n} \cdot E$ with $d(x, y)<\varepsilon$. Proof. This is a trivial variation of the above proof.

Proposition 5.3. Let $(A, d)$ be a non-zero $(\mathrm{F})$-algebra.

(i) Suppose that there exists a non-zero subset $S$ of $A$ such that $S \subset \overline{A \cdot S}$. Then property (B) holds for A.

(ii) Suppose that there exist a non-zero subset $S$ and $a \in A$ such that $S \subset \overline{a \cdot S}$. Then property (A) holds for $A$.

Proof. (i) Let $x_{0} \in S \backslash\{0\}$. Lemma 5.1 shows that there exist a sequence $\left(a_{n}\right)$ in $A$ and $y \in \lim _{\leftarrow} a_{1} \cdots a_{n} \cdot A$ with $d\left(x_{0}, y\right)<d\left(x_{0}, 0\right)$. In particular, $\lim _{\longleftarrow} a_{1} \cdots a_{n} \cdot A \neq\{0\}$.

(ii) This follows similarly from Lemma 5.2.

Corollary 5.4. Let $A$ be a non-zero $(\mathrm{F})$-algebra such that $\overline{A^{[2]}}=A$. Then condition (B) holds for A.

We note here the following curious fact as a consequence of the above and of Bohr's theorem on almost periodic functions.

Corollary 5.5. Let $E$ be a non-zero subspace of $M\left(\mathbb{R}^{+}\right)$, and let $f$ be any

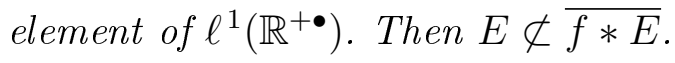

Proof. Assume towards a contradiction that there exist appropriate $E$ and $f$ such that $E \subset \overline{f * E}$. Then, by Proposition 5.3(ii), there exists a non-zero element $\mu$ in the set $\bigcap_{n=1}^{\infty} f^{* n} * M\left(\mathbb{R}^{+}\right)$. Thus $\alpha(\mu) \geq n \alpha(f)(n \in \mathbb{N})$, and so $\alpha(f)=0$. By [6, Theorem 4.7.55], there exists $z \in \Pi$ such that $(\mathcal{L} f)(z)=0$, and so $\mathcal{L} \mu$, which is an analytic function on $\Pi$, has a zero of infinite order at $z$. This implies that $\mu=0$, a contradiction.

The result follows.

We now use the above to extend the conditions characterizing class (III) of Esterle's classification that are given in [6, p. 578]. The existing classification in [6] supposes that algebra $R$ is a commutative, radical Banach 
algebra, but the equivalences hold true (with the same proofs) even if the radical algebra $R$ is not commutative and if it is an (F)-algebra, and so we state our result in this setting.

Theorem 5.6. Let $R$ be a non-zero, radical (F)-algebra. Then the following conditions on $R$ are equivalent:

(a) there is a non-zero element $a \in R$ such that $a \in \overline{R a}$;

(b) there is a sequence $\left(a_{n}\right)$ in $R$ such that $\varliminf_{\longleftarrow} a_{1} \cdots a_{n} \cdot R \neq\{0\}$;

(c) there is a sequence $\left(b_{n}\right)$ in $R^{\bullet}$ such that $b_{n} \in R b_{n+1}(n \in \mathbb{N})$;

(d) there is a strictly increasing sequence of principal left ideals in $R^{\sharp}$;

(e) there is a non-zero, left ideal $I$ in $R$ such that $I=\overline{R \cdot I}$;

(f) there is a non-zero, left ideal $J$ in $R$ such that $J=R \cdot J$;

(g) there is a non-zero subset $S$ in $R$ such that $S \subset \overline{R \cdot S}$.

Proof. That conditions (a), (b), (c), and (d) on $R$ are equivalent is established in [6], albeit in the case where $R$ is commutative. For the implication (a) $\Rightarrow(\mathrm{e})$, we can take $I=\overline{R a}$. Clearly $(\mathrm{c}) \Leftrightarrow(\mathrm{f}) \Rightarrow(\mathrm{e}) \Rightarrow(\mathrm{g})$. The implication $(\mathrm{g}) \Rightarrow$ (b) follows from Proposition 5.3(i).

We next extend the conditions characterizing class (IV) (of Esterle's classification) that are given in [6, p. 578].

Theorem 5.7. Let $R$ be a non-zero, commutative, radical (F)-algebra. Then the following conditions on $R$ are equivalent:

(a) there exist $a \in R$ and $x \in R \bullet$ with $a \in \overline{R a x}$;

(b) there exists $a \in R$ with $\lim _{\longleftarrow} a^{n} \cdot R \neq\{0\}$;

(c) there exist $a \in R$ and a non-zero subspace $S$ of $R$ such that $S=a S$;

(d) there exist $a \in R, y \in R^{\bullet}$, and a sequence $\left(p_{n}\right)$ of complex polynomials such that $y=\lim _{n \rightarrow \infty} a p_{n}(a) y$;

(e) there exist $a \in R$ and a non-zero ideal $I$ in $R$ such that $I=\overline{a I}$;

(f) there exist $a \in R$ and a non-zero subset $S$ in $R$ such that $S \subset \overline{a S}$.

Proof. That conditions (a), (b), (c), and (d) on $R$ are equivalent is established in [6]. For the implication $(\mathrm{a}) \Rightarrow(\mathrm{e})$, take $I=\overline{x R^{\sharp}}$. Clearly (e) $\Leftrightarrow(\mathrm{f})$. The implication (f) $\Rightarrow$ (b) follows from Proposition 5.3(ii).

In fact, all proofs of the implications between clauses (a) - (f) in the above theorem hold for a general (non-commutative) radical (F)-algebra, save for the implication $(\mathrm{a}) \Rightarrow(\mathrm{e})$. 
5.2. Non-nilpotent elements in radical (F)-algebras. In this subsection, we shall give various conditions for a (not necessarily commutative) radical (F)-algebra $R$ that are equivalent to the condition that $R$ have a non-nilpotent element $a$ with $a \in \overline{R a}$, following the spirit of [14] and [6, $\S 4.9]$. We are interested in this condition because, in the case where $R$ is a radical Banach algebra, it will imply the existence of an uncountable family of prime ideals in $R$ such that distinct primes in the family are incomparable with respect to inclusion; see $\S 5.3$, below.

Let $(X, d)$ be a metric space, and take $x \in X$ and $r>0$. Then we write $B(x, r)$ for the open ball with centre $x$ and radius $r$. The following lemma is essentially [6, Theorem 2.6.34], which also proves Grabiner's theorem that every nil (F)-algebra is nilpotent.

Lemma 5.8. Let $(A, d)$ be an $(\mathrm{F})$-algebra, let $S$ be a closed linear subspace of $A$, and take $n \in \mathbb{N}$. Suppose that there exist an element $a_{0} \in S$ and $r>0$ with the property that $a^{n}=0\left(a \in B\left(a_{0}, r\right) \cap S\right)$. Then $a^{n}=0$ for each $a \in S$.

Proof. For $a \in S$, define $F(\zeta)=\left(a_{0}+\zeta\left(a-a_{0}\right)\right)^{n}(\zeta \in \mathbb{C})$. Then $F$ is a polynomial with coefficients in $A$ such that $F$ vanishes on a neighbourhood of 0 in $\mathbb{C}$, and so $F=0$. Thus $a^{n}=F(1)=0$.

First, we need a technical lemma that is similar to Lemma 5.1.

Lemma 5.9. Let $(A, d)$ be an (F)-algebra, and let $S$ be a closed linear subspace of $A$ such that $S \subset \overline{A \cdot S}$. Then, for each non-nilpotent element $x_{0} \in S$ and $\varepsilon>0$, there exist a sequence $\left(a_{n}\right)$ in $A$ and a non-nilpotent element $y \in \lim _{\longleftarrow} a_{1} \cdots a_{n} A$ with $d\left(x_{0}, y\right)<\varepsilon$.

Proof. For $n \in \mathbb{N}$, set $U_{n}=\left\{a \in A: a^{n} \neq 0\right\}$, an open subset of $A$. Then we may suppose that each $U_{n}$ is dense in $A$, for otherwise Lemma 5.8 would imply that $A$ is nilpotent, and the lemma would be vacuous in this case. Define a continuous map $\theta_{n}: A^{n+1} \rightarrow A^{n}$ by

$$
\left(a_{1}, \ldots, a_{n}, a_{n+1}\right) \mapsto\left(a_{1}, \ldots, a_{n-1}, a_{n} a_{n+1}\right) .
$$

The composition $\mu_{n}:=\theta_{1} \circ \cdots \circ \theta_{n}$ is nothing but the multiplication map $A^{n+1} \rightarrow A$. Set

$$
E_{n}:=A^{n} \cap \mu_{n}^{-1}\left(U_{n}\right) .
$$

Then $E_{n}$ (with the product topology) is metrizable by a complete metric, and $\left(\left(E_{n}, \theta_{n}\right): n \in \mathbb{N}\right)$ is a projective sequence. Set

$$
X_{n}:=\left(A^{n-1} \times S\right) \cap E_{n},
$$


so that $X_{n}$ is closed in $E_{n}$.

We claim that $X_{n}$ is dense in $A^{n-1} \times S$. Indeed, otherwise, there would exist a non-empty, open subset $W$ of $A^{n-1} \times S$ with $W \cap \mu_{n}^{-1}\left(U_{n}\right)=\emptyset$. But this would then imply that $a^{n}=0$ for all $a \in \mu_{n}(W)$, which in turn would imply that $a^{n}=0$ for all $a \in \mu_{n}\left(A^{n-1} \times S\right)$, and again the lemma would be vacuous in this case.

This claim and the assumption that $S \subseteq \overline{A \cdot S}$ then imply that

$$
X_{n} \subset \overline{\theta_{n}\left(A^{n} \times S\right)} \subset \overline{\theta_{n}\left(X_{n+1}\right)} \quad(n \in \mathbb{N}),
$$

where the closures are taken in $A^{n}$. Thus Theorem 1.1 shows that $X_{1}$ is contained in the closure of $\underset{\lim }{\longleftarrow}\left(E_{n}, \theta_{n}\right)$, and the claim then shows that $S$ is contained in the closure of $\underset{\lim }{\longleftarrow}\left(E_{n}, \theta_{n}\right)$. This implies the result.

We now give our modified version of Theorem 5.6; it is related to [14, Corollary 3.5].

Theorem 5.10. Let $R$ be a non-zero, radical (F)-algebra. Then the following conditions on $R$ are equivalent:

(a) there is a non-nilpotent element $a \in R$ such that $a \in \overline{R a}$;

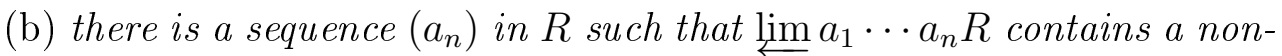
nilpotent element of $R$;

(c) there is a sequence $\left(b_{n}\right)$ in $R$ such that $b_{n} \in R b_{n+1}(n \in \mathbb{N})$ and $b_{1}$ is not nilpotent;

(d) there is a strictly increasing sequence of principal left ideals in $R^{\sharp}$, each of which is not nil;

(e) there is a strictly increasing sequence of principal left ideals in $R^{\sharp}$, each of which is not nilpotent;

(f) there is a left ideal $J$ in $R$ such that $J=R \cdot J$ and $J$ is not nil;

(g) there is a linear subspace $S$ in $R$ such that $S \subset \overline{R \cdot S}$ and $S$ is not nilpotent.

Proof. It is obvious that (c) $\Leftrightarrow$ (f), that $(\mathrm{d}) \Rightarrow(\mathrm{e})$, and that (a) $\Rightarrow$ (g) (with $S:=R^{\sharp} a$ ). The implications (b) $\Leftrightarrow(\mathrm{c})$ and $(\mathrm{c}) \Rightarrow(\mathrm{d})$ are also easy to see.

(e) $\Rightarrow(\mathrm{g})$ Let $\left(c_{n}\right)$ be a sequence in $R^{\sharp}$ such that $\left(R^{\sharp} c_{n}\right)$ is a strictly increasing sequence of principal left ideals in $R^{\sharp}$, each of which is not nil. Then $c_{n} \in R$ and $c_{n} \in R c_{n+1}$ for each $n \in \mathbb{N}$. Set $J=\bigcup\left\{R^{\sharp} c_{n}: n \in \mathbb{N}\right\}$, so that $J=\bigcup\left\{R c_{n}: n \in \mathbb{N}\right\}$. Then $J$ is a left ideal that is not nilpotent, and $J=R \cdot J \subset \overline{R \cdot J}$. 
(g) $\Rightarrow$ (b) By replacing $S$ by $\bar{S}$, we may suppose that $S$ is a closed linear subspace of $R$. Since $S$ is not nilpotent, there is an element $x_{0} \in S$ that is not nilpotent. Thus (b) follows from Lemma 5.9.

(c) $\Rightarrow$ (a) Take the sequence $\left(b_{n}\right)$ as specified, and set $J=\overline{\bigcup\left\{R b_{n}: n \in \mathbb{N}\right\}}$. Then $J$ is a closed, left ideal in $R$ and $J$ is not nil. Define

$$
S=\{x \in J: \overline{R x}=J\} .
$$

By a small variation of [14, Theorem 3.3], we see that $S$ contains a dense,

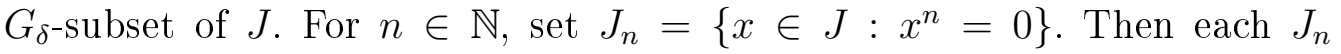
is a closed subset of $J$ with empty interior, and so, by Baire, there exists an element $a \in S \backslash \bigcup J_{n}$. The element $a$ is not nilpotent and is such that $a \in \overline{R a}$.

Corollary 5.11. Let $R$ be a non-zero, radical $(\mathrm{F})$-algebra with $R=\overline{R^{[2]}}$. Then there is a non-nilpotent element $a \in R$ such that $a \in \overline{R a}$.

Proof. Condition (g) of Theorem 5.11 holds, with $S=R$, and so condition (a) holds.

5.3. Prime ideals in radical convolution algebras. The family of prime ideals in a commutative Banach algebra plays a prominent role in automatic continuity theory; see [6], and see [31] for a further contribution. It is of interest to show the existence of 'large' families of incomparable primes.

It is easy to construct prime ideals in convolution algebras on $\mathbb{R}^{+}$. In this section, we shall use the results of the previous section and of [30] to show that a radical convolution algebra on $\mathbb{R}^{+}$must even have a continuum of incomparable prime ideals. We shall also discuss the discrete version of convolution algebras on sub-semigroups of $\mathbb{R}^{+}$.

A Fréchet algebra is an (F)-algebra whose topology is defined by a sequence of algebra seminorms [6, Definition 2.2.4].

Let $R$ be a commutative, radical Fréchet algebra containing a nonnilpotent element $a$ such that $a \in \overline{R a}$. The main result of [30], Corollary 3 , shows that $R$ then contains a continuum of incomparable prime ideals. This was proved as a consequence of [30, Theorem 2], which was stated for any (possibly non-commutative) radical Fréchet algebra $R$ that possesses such an element $a$. However, that theorem was not stated correctly, and we now rectify this error. The proof of [30, Theorem 2] does establish the following theorem. Here, $I^{(m)}$ denotes the $m$-fold Cartesian product of $I$ with itself for $m \in \mathbb{N}$. 
Theorem 5.12. Let $R$ be a radical Fréchet algebra. Suppose that $I$ is an ideal in $R$ that is not nilpotent and is such that

$$
I^{(m)}=\overline{R \cdot I^{(m)}} \quad(m \in \mathbb{N}) .
$$

Then there exists a sequence $\left(a_{n}\right)$ in I such that, for every Fréchet algebra A containing $R$ as a topological subalgebra,

$$
a_{j_{1}}^{k_{1}} \cdots a_{j_{n}}^{k_{n}} \notin a_{i_{1}} A+\cdots+a_{i_{m}} A
$$

for all finite sequences $\boldsymbol{i}=\left(i_{1}, \ldots, i_{m}\right), \boldsymbol{j}=\left(j_{1}, \ldots, j_{n}\right)$, and $\boldsymbol{k}=\left(k_{1}, \ldots, k_{n}\right)$ in $\mathbb{N}$ such that $\boldsymbol{i}$ and $\boldsymbol{j}$ are disjoint.

The new statement is as follows.

Proposition 5.13. Let $R$ be a radical Fréchet algebra, and suppose that a is a non-nilpotent, central element of $R$ with $a \in \overline{R a}$. Then $I:=\overline{R a}$ is an ideal in $R$ that is not nilpotent and such that I satisfies equation (5.1).

Proof. Set $I=\overline{R a}$, so that $I$ is an ideal in $R$ that is not nilpotent.

To prove that $I$ satisfies (5.1), take $m \in \mathbb{N}$ and open sets $W_{i}$ in $R$ for $i \in \mathbb{N}_{m}$ such that $W_{i} \cap I \neq \emptyset\left(i \in \mathbb{N}_{m}\right)$. Then there exist $x_{1}, \ldots, x_{m} \in R$ such that $x_{i} a \in W_{i}\left(i \in \mathbb{N}_{m}\right)$. Since $a$ is in the centre of $R$, it follows that $a x_{i}=x_{i} a \in W_{i}\left(i \in \mathbb{N}_{m}\right)$. Next, there exists $c \in R$ such that

$$
\operatorname{cax}_{i}=c x_{i} a \in W_{i}\left(i \in \mathbb{N}_{m}\right),
$$

and so

$$
R \cdot I^{(m)} \cap\left(W_{1} \times \cdots \times W_{m}\right) \neq \emptyset .
$$

Thus $I^{(m)} \subset \overline{R \cdot I^{(m)}}$, and hence $I$ satisfies (5.1).

Thus [30, Corollary 3], when combined with Corollary 5.11, gives the following theorem. Here $\mathfrak{c}$ denotes the continuum.

Theorem 5.14. Let $R$ be a non-zero, commutative, radical Fréchet algebra such that $R=\overline{R^{[2]}}$. Then there exists a family $\mathcal{F}=\left\{P_{\alpha}: \alpha \in \Lambda\right\}$ of prime ideals in $R$ such that $|\Lambda|=\mathfrak{c}$ and such that $P_{\alpha} \not \subset P_{\beta}$ whenever $\alpha, \beta \in \Lambda$ with $\alpha \neq \beta$.

The above theorem applies to any non-zero, commutative, radical Banach algebra $R$ that has an approximate identity, and in particular to the Volterra algebra $\mathcal{V}=\left(L^{1}(\mathbb{I}), \star\right)$ and the algebra $\left(C_{*, 0}(\mathbb{I}), \star\right)$. It also applies to algebras of the form $\left(L^{1}\left(\mathbb{R}^{+\bullet}, \omega\right), \star\right)$, where $\omega$ is a radical weight on $\mathbb{R}^{+\bullet}$; note that $\omega$ need not be bounded near the origin, and so this latter algebra may not have an approximate identity. Finally, we note that the theorem 
applies to Banach algebras $\left(\ell^{1}(S, \omega), \star\right)$ whenever $S$ is a dense, difference sub-semigroup of $\mathbb{R}^{+\bullet}$ and $\omega$ is a radical weight on $S$, and to the algebras $\left(\ell^{1}(S \cap \mathbb{I}), \star\right)$ whenever $S$ is a dense, difference sub-semigroup of $\mathbb{R}^{+\bullet}$.

\section{Summary}

We shall now summarize our attempts to establish counter-examples to a variety of implications between our specified factorization properties (I)(VII). We shall first briefly recall the definitions of the properties (I)-(VII); throughout $A$ is a commutative Banach algebra. Thus:

(I) $A$ has a bounded approximate identity;

(II) null sequences in $A$ factor;

(III) all pairs in $A$ factor;

(IV) $A$ factors;

(V) $A$ factors weakly;

(VI) $A$ factors projectively;

(VII) $A$ factors densely.

In the table, 'BFA' means 'Banach function algebra'; 'uniform' means 'maximal ideal in a uniform algebra'; 'CRBA' means 'commutative, radical Banach algebra'; 'sep' means 'separable'.

\begin{tabular}{|c|c|c|c|c|c|}
\hline Implication & BFA & sep BFA & uniform & sep uniform & sep CRBA \\
\hline \hline$(\mathrm{II}) \Rightarrow(\mathrm{I})$ & No, [36] & No, $[36]$ & No, Th. 3.4 & $?$ & No, [36] \\
\hline$(\mathrm{III}) \Rightarrow(\mathrm{II})$ & $?$ & $?$ & $?$ & $?$ & $?$ \\
\hline$(\mathrm{IV}) \Rightarrow(\mathrm{III})$ & No, $[29]$ & $?$ & No, $[29]$ & $?$ & $?$ \\
\hline$(\mathrm{IV}) \Rightarrow(\mathrm{I})$ & No, $[36]$ & No, $[36]$ & No, [18, 29] & $?$ & No, $[36]$ \\
\hline$(\mathrm{V}) \Rightarrow(\mathrm{IV})$ & No, $[36]$ & No, $[36]$ & $?$ & $?$ & $?$ \\
\hline$(\mathrm{VI}) \Rightarrow(\mathrm{V})$ & No, Ex. 1.13 & No, Ex. 1.13 & $?$ & $?$ & No, Ex. 1.13 \\
\hline$(\mathrm{VI}) \Rightarrow(\mathrm{III})$ & No, Ex. 1.13 & No, Ex. 1.13 & No, [29] & No, Ex. 1.18 & No, Ex. 1.13 \\
\hline$(\mathrm{VII}) \Rightarrow(\mathrm{VI})$ & No, Ex. 2.7 & No, Ex. 2.7 & No, Ex. 2.7 & No, Ex. 2.7 & No, Ex. 1.25 \\
\hline \hline
\end{tabular}

Acknowledgements. Some of this work was carried out whilst the first author was visiting the Victoria University of Wellington. He is grateful for generous hospitality and the financial support of the University and a London Mathematical Society travel grant.

The work was continued at a meeting of the first and second authors in Winnipeg, Manitoba. The second author thanks the London Mathematical Society for a travel grant. 
We are grateful to Alexander Izzo and Pamela Gorkin for some valuable discussions. We also thank the referee for valuable comments and the suggestion that our proofs of Lemmas 5.1 and 5.9 could be shortened by using the Mittag-Leffler theorem.

\section{REFERENCES}

[1] R. F. Basener, On rationally convex hulls, Trans. American Math. Soc., 182 (1973), $353-381$.

[2] M. Bożejko, Remark on Herz-Schur multipliers on free groups, Math. Ann., 258 (1981/82), 11-15.

[3] A Browder, Introduction to Function Algebras, Benjamin, New York, NY, 1969.

[4] B J. Cole, One point parts and the peak point conjecture, Ph.D. Thesis, Yale University, 1968.

[5] B. Cole, S. N. Ghosh, and A. J. Izzo, A hull with no non-trivial Gleason parts, Indiana University Mathematics J., 67 (2018), 739-752.

[6] H. G. Dales, Banach Algebras and Automatic Continuity, London Mathematical Society Monographs, New Series, Volume 24, The Clarendon Press, Oxford, 2000.

[7] H. G. Dales, F. K. Dashiell, A. T.-M. Lau, and D. Strauss, Banach Spaces of Continuous Functions as Dual Spaces, pp. xi + 260, Canadian Mathematical Society Books in Mathematics, Springer-Verlag, 2016.

[8] H. G. Dales and J. F. Feinstein, Banach function algebras with dense invertible group, Proc. American Math. Soc., 136 (2008), 1295-1304.

[9] H. G. Dales and A. Ülger, Approximate identities in Banach function algebras, Studia Math., 226 (2015), 155-187.

[10] H. G. Dales and A. Ülger, Pointwise approximate identities in Banach function algebras, Dissertationes Mathematicae (Rozprawy Matematyczne), submitted.

[11] A. Derighetti, Convolution Operators on Groups, Lecture Notes of the Unione Matematica Italiana, Volume 11, Springer, Heidelberg, 2011.

[12] P. G. Dixon, Factorization and unbounded approximate identities in Banach algebras, Math. Proc. Cambridge Philos. Soc., 107 (1990), 557-572.

[13] R. S. Doran and J. Wichmann, Approximate Identities and Factorization in Banach Modules, Lecture Notes in Mathematics, 768, Springer, 1979.

[14] J. R. Esterle, Elements for a classification of commutative radical Banach algebras Radical Banach algebras and automatic continuity, Lecture Notes in Mathematics, 975 (1983), 4-65.

[15] J. F. Feinstein, A non-trivial, strongly regular uniform algebra, J. London Math. Soc. (2), 45 (1992), 288-300.

[16] T. W. Gamelin, Uniform Algebras, Prentice-Hall, Englewood Cliffs, New Jersey, 1969.

[17] J. B. Garnett, Bounded Analytic Functions, Academic Press, New York, 1981.

[18] S. N. Ghosh and A. J. Izzo, in preparation.

[19] S. A. Grigoryan and T. V. Tonev, Shift-invariant Uniform Algebras on Groups, Monografie Matematyczne, Instytut Matematyczne, PAN, Volume 68, Birkhäuser, 2006.

[20] A. P. Hallstrom, On bounded point derivations and analytic capacity, J. Functional Analysis, 4 (1969), 153-165.

[21] C. S. Herz, Harmonic synthesis for subgroups, Ann. Inst. Fourier (Grenoble), 23 (1973), 91-123.

[22] K. Hoffman, Banach Spaces of Analytic Functions, Prentice Hall, Englewood Cliffs, New Jersey, 1962.

[23] M. Leinert, Faltungsoperatoren auf gewissen diskreten gruppen, Studia Math., 52 (1974), 149-158. 
[24] E. Kaniuth and A. T.-M. Lau, Fourier and Fourier-Stieltjes Algebras on Locally Compact Groups, Mathematical Surveys and Monographs, Volume 231, American Mathematical Society, Providence, Rhode Island, 2018.

[25] V. Losert, Some properties of groups without the property P1, Comment. Math. Helv., 54 (1979), 133-139.

[26] S. Morley, Extensions of uniform algebras, Banach J. Math. Anal., 13 (2019), 837863.

[27] A. G. O'Farrell, Density of parts of algebras on the plane, Trans. American Math. Soc., 196 (1971), 403-414.

[28] S. I. Ouzomgi, Factorization and bounded approximate identities for a class of convolution Banach algebras, Glasgow Math. J., 28 (1986), 211-214.

[29] S. I. Ouzomgi, A commutative Banach algebra with factorization of elements but not of pairs, Proc. American Math. Soc., 113 (1991), 435-441.

[30] H. L. Pham, Incomparable prime ideals in commutative radical Fréchet algebras, Proc. American Math. Soc. 137 (2009), 593-596.

[31] H. L. Pham, The kernels and continuity ideals of homomorphisms from $\mathcal{C}_{0}(\Omega)$, J. Australian Math. Soc., 88 (2010), 103-130.

[32] J.-P. Pier, Amenable Locally Compact Groups, Wiley, New York, 1984.

[33] E. L. Stout, The Theory of Uniform Algebras, Bogden and Quigley, Tarrytown-onHudson, New York, 1971.

[34] S. J. Sidney, Properties of the sequence of closed powers of a maximal ideal in a sup-norm algebra, Trans. American Math. Soc., 131 (1968), 128-148.

[35] J. Wermer, Bounded point derivations on certain Banach algebras, J. Functional Analysis, 1 (1967), 28-36.

[36] G. A. Willis, Examples of factorization without bounded approximate units, Proc. London Math. Soc. (3), 64 (1992), 602-624.

[37] G. A. Willis, Factorization in finite-codimensional ideals of group algebras, Proc. London Math. Soc. (3), 82 (2001), 676-700.

Department of Mathematics and Statistics, Fylde College, Lancaster UNIVERSITY, LANCASTER LA1 4YF, UK

Email address: g.dales@lancaster.ac.uk

School of Mathematical Sciences, University of Nottingham, NottingHAM, NG7 2RD, United Kingdom

Email address: Joel.Feinstein@nottingham.ac.uk

School of Mathematics and Statistics, Victoria University of WellingTon, Wellington 6140, New Zealand

Email address: hung.pham@vuw.ac.nz 